\title{
ABSTRACT CRYSTALS FOR QUANTUM BORCHERDS-BOZEC ALGEBRAS
}

\author{
ZHAOBING FAN, SEOK-JIN KANG, YOUNG ROCK KIM*, AND BOLUN TONG \\ ABSTRACT. In this paper, we develop the theory of abstract crystals for quantum Borcherds- \\ Bozec algebras. Our construction is different from the one given by Bozec. We further \\ prove the crystal embedding theorem and provide a characterization of $B(\infty)$ and $B(\lambda)$ \\ as its application, where $B(\infty)$ and $B(\lambda)$ are the crystals of the negative half part of the \\ quantum Borcherds-Bozec algebra $U_{q}(\mathfrak{g})$ and its irreducible highest weight module $V(\lambda)$, \\ respectively.
}

\section{Contents}

Introduction

1. The quantum Borcherds-Bozec algebras 4

2. Abstract crystals 10

3. Tensor product of crystals 17

4. Crystal embedding theorem 22

$\begin{array}{ll}\text { References } & 29\end{array}$

\section{INTRODUCTION}

The aim of this paper is to develop the theory of abstract crystals for quantum BorcherdsBozec algebras. To begin with, let us quickly review the term of Borcherds-Bozec algebras.

2010 Mathematics Subject Classification. 17B37, 17B67, 16G20.

Key words and phrases. quantum Borcherds-Bozec algebra, abstract crystals, crystal embedding theorem.

* Corresponding author. All authors contribute equally. 
The Cartan matrix of a semisimple Lie algebra is positive definite, where all of its diagonal entries are 2. In [Kac68, Moody68], Kac and Moody introduced a family of infinite dimensional Lie algebras by removing the condition of positive definitiveness, which are now called the Kac-Moody algebras. In [Bor88], Borcherds generalized the notion of KacMoody algebras by allowing the diagonal entries of the Cartan matrices to be non-positive. The Lie algebras and Cartan matrices thus obtained are called the Borcherds algebras and Borcherds-Cartan matrices, respectively. Moreover, the Borcherds algebras are generated by positive and negative simple root vectors. Associated to Borcherds-Cartan matrices, the Borcherds-Bozec algebras is a further generalization of Kac-Moody algebras, which are generated by higher degree positive and negative simple root vectors.

The quantum Borcherds algebras (or quantum generalized Kac-Moody algebras) were introduced by Kang [K95], as a quantum deformation of the universal enveloping algebras of Borcherds algebras. The quantum Borcherds-Bozec algebras were introduced by Bozec in his research of perverse sheaves theory for quivers with loops [B15, B16, BSV16]. They are a further generalization of quantum Borcherds algebras. More precisely, for each imaginary index $i \in I^{\mathrm{im}}$, i.e., those $i \in I$ satisfying $a_{i i} \leq 0$ in the Cartan matrix, there are infinitely many generators $e_{i l}, f_{i l}\left(l \in \mathbb{Z}_{>0}\right)$, whose degrees are $l$ multiples of $\alpha_{i}$ and $-\alpha_{i}$. The commutation relations between them are rather complicated (cf. [FKKT20]). But, thanks to Bozec, there exists a set of primitive generators in quantum BorcherdsBozec algebras with better properties and simpler commutation relations. This allows us to generalize the theories of quantum groups to quantum Borcherds-Bozec algebras.

The canonical basis theory was first introduced by Lusztig in the ADE case in [L90], arising from his geometric construction of the negative part of the quantum groups, and it has been generalized to general cases by Lusztig [L91, L10]. Meanwhile, Kashiwara constructed crystal base and global base for quantum groups associated with symmetrizable Kac-Moody algebras in an algebraic way [Kas90, Kas91]. In [GL93], Grojnowski and Lusztig proved that the global crystal base coincides with the canonical base introduced by Lusztig in [L90]. Since then, the crystal basis theory has become one of the most central themes in combinatorial and geometric representation theory of quantum groups. In [JKK05], Jeong, Kang and Kashiwara developed the crystal basis theory for quantum 
Borcherds algebras. Later on, together with Shin, they further introduced the notion of abstract crystals for quantum Borcherds algebras and investigated their fundamental properties [JKKS07].

In [B16], Bozec studied the crystal basis theory for quantum Borcherds-Bozec algebra $U_{q}(\mathfrak{g})$. He defined the crystal basis $(L(\infty), B(\infty))$ for $U_{q}^{-}(\mathfrak{g})$ and $(L(\lambda), B(\lambda))$ for $V(\lambda)$, respectively, where $V(\lambda)$ is the irreducible highest weight $U_{q}(\mathfrak{g})$-module with a dominant integral highest weight $\lambda$. He also constructed generalized crystals and their geometric realization for $U_{q}^{-}(\mathfrak{g})$ and $V(\lambda)$ based on the theory of perverse sheaves on Lusztig's and Nakajima's quiver varieties associated with quivers with loops (cf. [KS97, S02, KKS09, KKS12]).

In this paper, we develop the theory of abstract crystals for quantum Borcherds-Bozec algebras. Our construction is different from the one given in [B16], where Bozec followed the framework of [JKK05]. Instead, we take the approach given in [JKKS07], which is simpler and more natural. We provide the abstract crystal structure on $B(\infty)$ and $B(\lambda)$ following our definition. Moreover, we define the tensor products of abstract crystals which are shown to be abstract crystals. We also show that the associativity law holds for the tensor products of abstract crystals.

Furthermore, we prove the crystal embedding theorem, which yields a procedure to determine the structure of the crystal $B(\infty)$ in term of elementary crystals. We provide a characterization of $B(\infty)$ and $B(\lambda)$ as an application of the crystal embedding theorem. The characterization of $B(\lambda)$ in the current paper is different from the one given by Bozec in [B16], where the method of Joseph in [J95] was employed.

Our theory of abstract crystals can be applied to various general settings. For example, Bozec's geometric construction of the crystals $B(\infty)$ and $B(\lambda)$ can be reproduced through our approach. Our construction of $B(\infty)$ and $B(\lambda)$ can also be used directly to the theory of (dual) perfect bases for quantum Borcherds-Bozec algebras. Moreover, our theory will play a crucial role in the categorification of quantum Borcherds-Bozec algebras and their highest weight modules via Khovanov-Lauda-Rouquier algebras and their cyclotomic quotients. Currently, we are working on these problems as well as other applications. We 
believe that our work on abstract crystals will provide a strong algebraic foundation for a wide variety of interesting developments in combinatorial and geometric representation theory of quantum Borcherds-Bozec algebras.

This paper is organized as follows. In Section 1, we review some of the basic facts on quantum Borcherds-Bozec algebras. Under the assumption (1.1), the presentation of quantum Borcherds-Bozec algebras becomes simpler. In Section 2, we define the notion of abstract crystals and investigate their fundamental properties. In Section 3, We define the tensor products of abstract crystals having all the desired properties. In Section 4, we prove the crystal embedding theorem. As an application, we provide a characterization of $B(\infty)$ and $B(\lambda)$.

\section{Acknowledgements.}

Z. Fan was partially supported by the NSF of China grant 11671108, the NSF of Heilongjiang Province grant JQ2020A001, and the Fundamental Research Funds for the central universities. S.-J. Kang was supported by Hankuk University of Foreign Studies Research Fund. Y. R. Kim was supported by the Basic Science Research Program of the NRF (Korea) under grant No. 2015R1D1A1A01059643. S.-J. Kang would like to express his sincere gratitude to Harbin Engineering University for their hospitality during his visit in July and November, 2019.

\section{The quantum Borcherds-Bozec Algebras}

Let $I$ be a finite or countably infinite index set. An integer-valued matrix $A=\left(a_{i j}\right)_{i, j \in I}$ is called an even symmetrizable Borcherds-Cartan matrix if it satisfies the following conditions:

(i) $a_{i i}=2,0,-2,-4, \ldots$,

(ii) $a_{i j} \in \mathbb{Z}_{\leq 0}$ for $i \neq j$,

(iii) there is a diagonal matrix $D=\operatorname{diag}\left(r_{i} \in \mathbb{Z}_{>0} \mid i \in I\right)$ such that $D A$ is symmetric. 
Let $I^{\mathrm{re}}:=\left\{i \in I \mid a_{i i}=2\right\}, I^{\mathrm{im}}:=\left\{i \in I \mid a_{i i} \leq 0\right\}$, and $I^{\text {iso }}:=\left\{i \in I \mid a_{i i}=0\right\}$. The elements of $I^{\mathrm{re}}$ (resp. $I^{\mathrm{im}}, I^{\mathrm{iso}}$ ) are called real indices (resp. imaginary indices, isotropic indices).

\section{A Borcherds-Cartan datum consists of}

(a) an even symmtrizable Borcherds-Cartan matrix,

(b) a free abelian group $P$, the weight lattice,

(c) $\Pi=\left\{\alpha_{i} \in P \mid i \in I\right\}$, the set of simple roots,

(d) $P^{\vee}:=\operatorname{Hom}_{\mathbb{Z}}(P, \mathbb{Z})$, the dual weight lattice,

(e) $\Pi^{\vee}=\left\{h_{i} \mid i \in I\right\}$, the set of simple coroots

satisfying the following conditions:

(i) $\left\langle h_{i}, \alpha_{j}\right\rangle=a_{i j}$ for all $i, j \in I$,

(ii) $\Pi$ is linearly independent over $\mathbb{Z}$,

(iii) for each $i \in I$, there is an element $\Lambda_{i} \in P$, called the fundamental weights, such that

$$
\left\langle h_{j}, \Lambda_{i}\right\rangle=\delta_{i j} \quad \text { for all } j \in I \text {. }
$$

We denote by

$$
P^{+}:=\left\{\lambda \in P \mid\left\langle h_{i}, \lambda\right\rangle \geq 0 \text { for all } i \in I\right\},
$$

the set of dominant integral weights. The free abelian group $Q:=\bigoplus_{i \in I} \mathbb{Z} \alpha_{i}$ is called the root lattice. Set $Q^{+}=\sum_{i \in I} \mathbb{Z}_{\geq 0} \alpha_{i}$ and $Q^{-}=-Q^{+}$. For $\beta=\sum k_{i} \alpha_{i} \in Q^{+}$, we define its height to be ht $(\beta):=\sum k_{i}$.

Set $\mathfrak{h}=\mathbb{Q} \otimes_{\mathbb{Z}} P^{\vee}$, the Cartan subalgebra. Since $A$ is symmetrizable and $\Pi$ is linearly independent, there is a non-degenerate symmetric bilinear form $($,$) on \mathfrak{h}^{*}$ satisfying

$$
\left(\alpha_{i}, \lambda\right)=r_{i}\left\langle h_{i}, \lambda\right\rangle \text { for all } i \in I, \lambda \in \mathfrak{h}^{*} .
$$

For $i \in I^{\text {re }}$, we define the simple reflection $\omega_{i} \in G L\left(\mathfrak{h}^{*}\right)$ by

$$
\omega_{i}(\lambda)=\lambda-\left\langle h_{i}, \lambda\right\rangle \alpha_{i} \text { for } \lambda \in \mathfrak{h}^{*} .
$$

The subgroup $W$ of $G L\left(\mathfrak{h}^{*}\right)$ generated by $\omega_{i}\left(i \in I^{\text {re }}\right)$ is called the Weyl group. One can easily verify that the symmetric bilinear form $($,$) is W$-invariant. 
Let $I^{\infty}:=\left(I^{\mathrm{re}} \times\{1\}\right) \cup\left(I^{\mathrm{im}} \times \mathbb{Z}_{>0}\right)$. For simplicity, we shall often write $i$ instead of $(i, 1)$ for $i \in I^{\text {re }}$. Let $q$ be an indeterminate and set

$$
q_{i}=q^{r_{i}} \text { and } q_{(i)}=q^{\frac{\left(\alpha_{i}, \alpha_{i}\right)}{2}} .
$$

Note that $q_{i}=q_{(i)}$ if $i \in I^{\text {re }}$. For each $i \in I^{\text {re }}$ and $n \in \mathbb{Z}_{\geq 0}$, we define

$$
[n]_{i}=\frac{q_{i}^{n}-q_{i}^{-n}}{q_{i}-q_{i}^{-1}}, \quad[n]_{i} !=\prod_{k=1}^{n}[k]_{i} \quad \text { and } \quad\left[\begin{array}{l}
n \\
k
\end{array}\right]_{i}=\frac{[n]_{i} !}{[k]_{i} ![n-k]_{i} !} .
$$

Let $\mathscr{F}$ be the free associative algebra over $\mathbb{Q}(q)$ generated by the symbols $f_{i l}$ for $(i, l) \in$ $I^{\infty}$. By setting $\operatorname{deg} f_{i l}=-l \alpha_{i}, \mathscr{F}$ becomes a $Q^{-}$-graded algebra. For any homogeneous element $u$ in $\mathscr{F}$, we denote by $|u|$ the degree of $u$, and for any $A \subseteq Q^{-}$, we set $\mathscr{F}_{A}=\{x \in$ $\mathscr{F}|| x \mid \in A\}$.

We define a twisted multiplication on $\mathscr{F} \otimes \mathscr{F}$ by

$$
\left(x_{1} \otimes x_{2}\right)\left(y_{1} \otimes y_{2}\right)=q^{-\left(\left|x_{2}\right|,\left|y_{1}\right|\right)} x_{1} y_{1} \otimes x_{2} y_{2},
$$

and a comultiplication $\delta: \mathscr{F} \rightarrow \mathscr{F} \otimes \mathscr{F}$ by

$$
\delta\left(f_{i l}\right)=\sum_{m+n=l} q_{(i)}^{-m n} f_{i m} \otimes f_{i n} \text { for }(i, l) \in I^{\infty} .
$$

Here $f_{i 0}=1$ and $f_{i l}=0$ for $l<0$ by convention.

Proposition 1.1. [B15, B16] For any family $\nu=\left(\nu_{i l}\right)_{(i, l) \in I^{\infty}}$ of non-zero elements in $\mathbb{Q}(q)$, there exists a symmetric bilinear form $(,)_{L}: \mathscr{F} \times \mathscr{F} \rightarrow \mathbb{Q}(q)$ such that

(a) $(x, y)_{L}=0$ if $|x| \neq|y|$.

(b) $(1,1)_{L}=1$.

(c) $\left(f_{i l}, f_{i l}\right)_{L}=\nu_{i l}$ for all $(i, l) \in I^{\infty}$.

(d) $(x, y z)_{L}=(\delta(x), y \otimes z)_{L}$ for all $x, y, z \in \mathscr{F}$,

where $\left(x_{1} \otimes x_{2}, y_{1} \otimes y_{2}\right)_{L}=\left(x_{1}, y_{1}\right)_{L}\left(x_{2}, y_{2}\right)_{L}$ for any $x_{1}, x_{2}, y_{1}, y_{2} \in \mathscr{F}$.

From now on, we will assume that

$$
\nu_{i l} \in 1+q \mathbb{Z}_{\geq 0}[[q]] \text { for all }(i, l) \in I^{\infty}
$$


Under the assumption (1.1), the radical of the bilinear form $(,)_{L}$ is generated by

$$
\begin{aligned}
& \sum_{k=0}^{1-l a_{i j}}(-1)^{k}\left[\begin{array}{c}
1-l a_{i j} \\
k
\end{array}\right]_{i} f_{i}{ }^{1-l a_{i j}-k} f_{j l} f_{i}^{k}=0 \text { for } i \in I^{\mathrm{re}}, i \neq(j, l), \\
& f_{i k} f_{j l}-f_{j l} f_{i k}=0 \text { for } a_{i j}=0 .
\end{aligned}
$$

Let $U^{\leq 0}$ be the associative algebra over $\mathbb{Q}(q)$ with 1 generated by $f_{i l}\left((i, l) \in I^{\infty}\right)$ and $q^{h}\left(h \in P^{\vee}\right)$ with the defining relations (1.2) and

$$
\begin{aligned}
& q^{0}=\mathbf{1}, \quad q^{h} q^{h^{\prime}}=q^{h+h^{\prime}} \quad \text { for } h, h^{\prime} \in P^{\vee}, \\
& q^{h} f_{j l} q^{-h}=q^{-l\left\langle h, \alpha_{j}\right\rangle} f_{j l} \text { for } h \in P^{\vee},(j, l) \in I^{\infty} .
\end{aligned}
$$

The comultiplication $\delta$ induces a well-defined comultiplication $\Delta: U^{\leq 0} \rightarrow U^{\leq 0} \otimes U \leq 0$ given by

$$
\begin{aligned}
& \Delta\left(q^{h}\right)=q^{h} \otimes q^{h} \\
& \Delta\left(f_{i l}\right)=\sum_{m+n=l} q_{(i)}^{-m n} f_{i m} K_{i}^{n} \otimes f_{i n} .
\end{aligned}
$$

By the Drinfeld double process, we have the following definition for quantum BorcherdsBozec algebra.

Definition 1.2. The quantum Borcherds-Bozec algebra $U_{q}(\mathfrak{g})$ associated with a BorcherdsCartan datum $\left(A, P, \Pi, P^{\vee}, \Pi^{\vee}\right)$ is the associative algebra over $\mathbb{Q}(q)$ with $\mathbf{1}$ generated by 
the elements $q^{h}\left(h \in P^{\vee}\right)$ and $e_{i l}, f_{i l}\left((i, l) \in I^{\infty}\right)$ with the following defining relations:

$$
\begin{aligned}
& q^{0}=1, \quad q^{h} q^{h^{\prime}}=q^{h+h^{\prime}} \text { for } h, h^{\prime} \in P^{\vee}, \\
& q^{h} e_{j l} q^{-h}=q^{l\left\langle h, \alpha_{j}\right\rangle} e_{j l}, \quad q^{h} f_{j l} q^{-h}=q^{-l\left\langle h, \alpha_{j}\right\rangle} f_{j l} \text { for } h \in P^{\vee},(j, l) \in I^{\infty}, \\
& e_{i k} f_{j l}-f_{j l} e_{i k}=0 \text { for } i \neq j, \\
& \sum_{\substack{m+n=k \\
n+s=l}} q_{(i)}^{n(m-s)} \nu_{i n} e_{i s} f_{i m} K_{i}^{-n}=\sum_{\substack{m+n=k \\
n+s=l}} q_{(i)}^{-n(m-s)} \nu_{i n} f_{i m} e_{i s} K_{i}^{n} \text { for }(i, l),(i, k) \in I^{\infty}, \\
& \sum_{k=0}^{1-l a_{i j}}(-1)^{k}\left[\begin{array}{c}
1-l a_{i j} \\
k
\end{array}\right]_{i} e^{1-l a_{i j}-k} e_{j l} e_{i}^{k}=0 \text { for } i \in I^{\mathrm{re}}, i \neq(j, l), \\
& \sum_{k=0}^{1-l a_{i j}}(-1)^{k}\left[\begin{array}{c}
1-l a_{i j} \\
k
\end{array}\right]_{i} f_{i}^{1-l a_{i j}-k} f_{j l} f_{i}^{k}=0 \text { for } i \in I^{\mathrm{re}}, i \neq(j, l), \\
& e_{i k} e_{j l}-e_{j l} e_{i k}=f_{i k} f_{j l}-f_{j l} f_{i k}=0 \text { for } a_{i j}=0 .
\end{aligned}
$$

Here $K_{i}=q_{i}^{h_{i}}(i \in I)$. We extend the grading by setting $\left|q^{h}\right|=0$ and $\left|e_{i l}\right|=l \alpha_{i}$.

Remark 1.3. Our presentation of $U_{q}(\mathfrak{g})$ is simpler than the ones in [B15, B16, BSV16] thanks to the explicit commutation relations among the generators given in [FKKT20, Appendix A].

The comultiplication on $U^{\leq 0}$ can be extended to the comultiplication $\Delta: U_{q}(\mathfrak{g}) \rightarrow$ $U_{q}(\mathfrak{g}) \otimes U_{q}(\mathfrak{g})$ given by

$$
\begin{aligned}
& \Delta\left(q^{h}\right)=q^{h} \otimes q^{h} \\
& \Delta\left(e_{i l}\right)=\sum_{m+n=l} q_{(i)}^{m n} e_{i m} \otimes K_{i}^{-m} e_{i n} \\
& \Delta\left(f_{i l}\right)=\sum_{m+n=l} q_{(i)}^{-m n} f_{i m} K_{i}^{n} \otimes f_{i n} .
\end{aligned}
$$

Let $U^{+}$(resp. $U^{-}$) be the subalgebra of $U_{q}(\mathfrak{g})$ generated by $e_{i l}$ (resp. $f_{i l}$ ) for $(i, l) \in I^{\infty}$, and $U^{0}$ the subalgebra of $U_{q}(\mathfrak{g})$ generated by $q^{h}$ for $h \in P^{\vee}$. Then the quantum BorcherdsBozec algebra $U_{q}(\mathfrak{g})$ has the following triangular decomposition

$$
U_{q}(\mathfrak{g}) \cong U^{-} \otimes U^{0} \otimes U^{+} .
$$


Let $\omega: U_{q}(\mathfrak{g}) \rightarrow U_{q}(\mathfrak{g})$ be the involution defined by

$$
\omega\left(q^{h}\right)=q^{-h}, \omega\left(e_{i l}\right)=f_{i l}, \omega\left(f_{i l}\right)=e_{i l} \text { for } h \in P^{\vee},(i, l) \in I^{\infty},
$$

and define a $\mathbb{Q}$-algebra involution on $U_{q}(\mathfrak{g})$, called the bar involution, by

$$
\bar{e}_{i l}=e_{i l}, \bar{f}_{i l}=f_{i l}, \bar{q}^{h}=q^{-h}, \bar{q}=q^{-1} \text { for } h \in P^{\vee},(i, l) \in I^{\infty} .
$$

The following proposition provides a set of primitive generators for $U_{q}(\mathfrak{g})$.

Proposition 1.4. [B15, B16] For any $i \in I^{\text {im }}$ and $l \geq 1$, there exist unique elements $t_{i l} \in U_{-l \alpha_{i}}^{-}$and $s_{i l}=\omega\left(t_{i l}\right)$ such that

(1) $\mathbb{Q}(q)\left\langle f_{i l} \mid l \geq 1\right\rangle=\mathbb{Q}(q)\left\langle t_{i l} \mid l \geq 1\right\rangle$ and $\mathbb{Q}(q)\left\langle e_{i l} \mid l \geq 1\right\rangle=\mathbb{Q}(q)\left\langle s_{i l} \mid l \geq 1\right\rangle$,

(2) $\left(t_{i l}, z\right)_{L}=0$ for all $z \in \mathbb{Q}(q)\left\langle f_{i 1}, \cdots, f_{i, l-1}\right\rangle$, $\left(s_{i l}, z\right)_{L}=0$ for all $z \in \mathbb{Q}(q)\left\langle e_{i 1}, \cdots, e_{i, l-1}\right\rangle$,

(3) $t_{i l}-f_{i l} \in \mathbb{Q}(q)\left\langle f_{i k} \mid k<l\right\rangle$ and $s_{i l}-e_{i l} \in \mathbb{Q}(q)\left\langle e_{i k} \mid k<l\right\rangle$,

(4) $\bar{t}_{i l}=t_{i l}, \quad \bar{s}_{i l}=s_{i l}$,

(5) $\delta\left(t_{i l}\right)=t_{i l} \otimes 1+1 \otimes t_{i l}, \delta\left(s_{i l}\right)=s_{i l} \otimes 1+1 \otimes s_{i l}$,

(6) $\Delta\left(t_{i l}\right)=t_{i l} \otimes 1+K_{i}^{l} \otimes t_{i l}, \Delta\left(s_{i l}\right)=s_{i l} \otimes K_{i}^{-l}+1 \otimes s_{i l}$,

Set $\tau_{i l}=\left(t_{i l}, t_{i l}\right)_{L}=\left(s_{i l}, s_{i l}\right)_{L}$. We have the following commutation relations in $U_{q}(\mathfrak{g})$

$$
s_{i l} t_{j k}-t_{j k} s_{i l}=\delta_{i j} \delta_{l k} \tau_{i l}\left(K_{i}^{l}-K_{i}^{-l}\right) .
$$

For $i \in I^{\text {im }} \backslash I^{\text {iso }}$ (resp. $i \in I^{\text {iso }}$ ), denote by $\mathcal{C}_{i, l}$ the set of compositions (resp. partitions) of $l$, and set $\mathcal{C}_{i}=\bigsqcup_{l \geq 0} \mathcal{C}_{i, l}$. For $i \in I^{\text {re }}$, we put $\mathcal{C}_{i, l}=\{(l)\}$.

If $i \in I^{\mathrm{im}}$ and $\mathbf{c}=\left(c_{1}, \cdots, c_{r}\right) \in \mathcal{C}_{i, l}$, we write $|\mathbf{c}|=l$ and define

$$
t_{i, \mathbf{c}}=t_{i c_{1}} \cdots t_{i c_{r}}, \quad s_{i, \mathbf{c}}=s_{i c_{1}} \cdots s_{i c_{r}} \text { and } \tau_{i, \mathbf{c}}=\tau_{i c_{1}} \cdots \tau_{i c_{r}} .
$$

Note that $\left\{t_{i, \mathbf{c}} \mid \mathbf{c} \in \mathcal{C}_{i, l}\right\}$ forms a basis of $U_{-l \alpha_{i}}^{-}$.

For each $(i, l) \in I^{\infty}$, we define the linear map $e_{i l}^{\prime}: U^{-} \rightarrow U^{-}$by

$$
e_{i l}^{\prime}(1)=0, e_{i l}^{\prime}\left(t_{j k}\right)=\delta_{i j} \delta_{l k} \text { and } e_{i l}^{\prime}(x y)=e_{i l}^{\prime}(x) y+q^{l\left(|x|, \alpha_{i}\right)} x e_{i l}^{\prime}(y) .
$$

In [B16], Bozec used the operators $e_{i l}^{\prime}$ to define the Kashiwara operators on $U^{-}$. 


\section{Abstract CRYstals}

In this section, we develop the theory of abstract crystals for quantum Borcherds-Bozec algebras. We would like to point out that we follow the outline given in [JKKS07], not in [JKK05].

Definition 2.1. Let $U_{q}(\mathfrak{g})$ be the quantum Borcherds-Bozec algebra associated with a given Borcherds-Cartan datum $\left(A, P, P^{\vee}, \Pi, \Pi^{\vee}\right)$. An abstract $U_{q}(\mathfrak{g})$-crystal or simply a crystal is a set $B$ together with the maps wt: $B \rightarrow P, \widetilde{e}_{i l}, \widetilde{f}_{i l}: B \rightarrow B \sqcup\{0\}\left((i, l) \in I^{\infty}\right)$ and $\epsilon_{i}, \phi_{i}: B \rightarrow \mathbb{Z} \sqcup\{-\infty\}(i \in I)$ satisfying the following conditions:

(i) $\operatorname{wt}\left(\widetilde{e}_{i, l} b\right)=\operatorname{wt}(b)+l \alpha_{i}$ if $\widetilde{e}_{i l} b \neq 0$.

(ii) $\operatorname{wt}\left(\widetilde{f}_{i, l} b\right)=\operatorname{wt}(b)-l \alpha_{i}$ if $\tilde{f}_{i l} b \neq 0$.

(iii) For any $i \in I$ and $b \in B, \phi_{i}(b)=\epsilon_{i}(b)+\left\langle h_{i}, \operatorname{wt}(b)\right\rangle$.

(iv) For any $(i, l) \in I^{\infty}$ and $b, b^{\prime} \in B, \widetilde{f}_{i l} b=b^{\prime}$ if and only if $b=\widetilde{e}_{i l} b^{\prime}$.

(v) For any $i \in I^{\text {re }}$ and $b \in B$, we have

(a) $\epsilon_{i}\left(\widetilde{e}_{i} b\right)=\epsilon_{i}(b)-1, \phi_{i}\left(\widetilde{e}_{i} b\right)=\phi_{i}(b)+1$ if $\widetilde{e}_{i} b \neq 0$,

(b) $\epsilon_{i}\left(\tilde{f}_{i} b\right)=\epsilon_{i}(b)+1, \phi_{i}\left(\tilde{f}_{i} b\right)=\phi_{i}(b)-1$ if $\tilde{f}_{i} b \neq 0$, where $\widetilde{e}_{i}=\widetilde{e}_{i 1}$ and $\widetilde{f}_{i}=\widetilde{f}_{i 1}$.

(vi) For any $i \in I^{\mathrm{im}}, l>0$ and $b \in B$, we have

(a) $\epsilon_{i}\left(\widetilde{e}_{i l} b\right)=\epsilon_{i}(b), \phi_{i}\left(\widetilde{e}_{i l} b\right)=\phi_{i}(b)+l a_{i i}$ if $\widetilde{e}_{i l} b \neq 0$,

(b) $\epsilon_{i}\left(\tilde{f}_{i l} b\right)=\epsilon_{i}(b), \phi_{i}\left(\tilde{f}_{i l} b\right)=\phi_{i}(b)-l a_{i i}$ if $\tilde{f}_{i l} b \neq 0$.

(vii) For any $(i, l) \in I^{\infty}$ and $b \in B$ such that $\phi_{i}(b)=-\infty$, we have $\widetilde{e}_{i l} b=\widetilde{f}_{i l} b=0$.

Let $B$ be an abstract crystal. For $b, b^{\prime} \in B$ and $(i, l) \in I^{\infty}$, by the condition (iv), we have $\widetilde{f}_{i l} b=b^{\prime}$ if and only if $\widetilde{e}_{i l} b^{\prime}=b$. In this case,we draw a labelled arrow $b \stackrel{(i, l)}{\longrightarrow} b^{\prime}$. The directed graph thus obtained is called the crystal graph of $B$.

Definition 2.2. Let $B_{1}$ and $B_{2}$ be abstract crystals. A map $\psi: B_{1} \rightarrow B_{2}$ is called a morphism of crystals or a crystal morphism if it satisfies the following conditions:

(i) for $b \in B_{1}$ and $i \in I$, we have $\operatorname{wt}(\psi(b))=\operatorname{wt}(b), \epsilon_{i}(\psi(b))=\epsilon_{i}(b), \phi_{i}(\psi(b))=\phi_{i}(b)$,

(ii) for all $b \in B_{1},(i, l) \in I^{\infty}$, if $\widetilde{f}_{i l} b \in B_{1}$, then $\psi\left(\widetilde{f}_{i l} b\right)=\widetilde{f}_{i l} \psi(b)$. 


\section{Remark 2.3.}

(a) If $b \in B_{1}$ and $\widetilde{e}_{i l} b \in B_{1}$, one can deduce $\psi\left(\widetilde{e}_{i l} b\right)=\widetilde{e}_{i l} \psi(b)$.

(b) If a crystal morphism $\psi$ is a bijection, then $\psi^{-1}$ is also a crystal morphism. In particular, both $\psi$ and $\psi^{-1}$ commute with $\widetilde{e}_{i l}$ 's.

Definition 2.4. Let $\psi: B_{1} \rightarrow B_{2}$ be a morphism of crystals.

(a) $\psi$ is called a strict morphism if

$$
\psi\left(\widetilde{e}_{i l} b\right)=\widetilde{e}_{i l}(\psi(b)), \quad \psi\left(\widetilde{f}_{i l} b\right)=\widetilde{f}_{i l}(\psi(b))
$$

for all $b \in B_{1},(i, l) \in I^{\infty}$. Here, we understand $\psi(0)=0$.

(b) $\psi$ is called a crystal embedding if the underlying map $\psi: B_{1} \rightarrow B_{2}$ is injective. In this case, $B_{1}$ is called a subcrystal of $B_{2}$. If $\psi$ is a strict embedding, $B_{1}$ is called a full subcrystal of $B_{2}$.

(c) $\psi$ is called an isomorphism if it is a bijection.

In the following two examples, we recall the crystals $B(\infty)$ and $B(\lambda)\left(\lambda \in P^{+}\right)$constructed in [B16].

\section{Example 2.5.}

Let $u \in U^{-}$and $(i, l) \in I^{\infty}$.

If $i \in I^{\mathrm{re}}$, in [Kas91], Kashiwara proved that $u$ can be uniquely written as

$$
u=\sum_{k \geq 0} f_{i}^{(k)} u_{k}
$$

where $f_{i}^{(k)}=f_{i}^{k} /[k]_{i}$ ! and $e_{i}^{\prime} u_{k}=0$ for all $k \geq 0$. In this case, the Kashiwara operators are defined by

$$
\widetilde{e}_{i} u=\sum_{k \geq 1} f_{i}^{(k-1)} u_{k}, \quad \widetilde{f}_{i} u=\sum_{k \geq 0} f_{i}^{(k+1)} u_{k} .
$$

If $i \in I^{\text {im }} \backslash I^{\text {iso }}$, in [B16], Bozec showed that $u$ can be written uniquely as

$$
u=\sum_{\mathbf{c} \in \mathcal{C}_{i}} t_{i, \mathbf{c}} u_{\mathbf{c}}
$$


where $\mathbf{c}=\left(c_{1}, \ldots, c_{r}\right)$ is a composition in $\mathcal{C}_{i}$ and $e_{i l}^{\prime} u_{\mathbf{c}}=0$ for all $l \geq 1$. In this case, the Kashiwara operators are defined by

$$
\widetilde{e}_{i l} u=\sum_{\mathbf{c}: c_{1}=l} t_{i, \mathbf{c} \backslash c_{1}} u_{\mathbf{c}}, \quad \widetilde{f}_{i l} u=\sum_{\mathbf{c} \in \mathcal{C}_{i}} t_{i,(l, \mathbf{c})} u_{\mathbf{c}}
$$

If $i \in I^{\text {iso }}, u$ can be written uniquely as

$$
u=\sum_{\mathbf{c} \in \mathcal{C}_{i}} t_{i, \mathbf{c}} u_{\mathbf{c}}
$$

where $\mathbf{c}=\left(c_{1}, \ldots, c_{r}\right)$ is a partition in $\mathcal{C}_{i}$ and $e_{i l}^{\prime} u_{\mathbf{c}}=0$ for all $l \geq 1$. In this case, the Kashiwara operators are defined by

$$
\widetilde{e}_{i l} u=\sum_{\mathbf{c} \in \mathcal{C}_{i}} \sqrt{\frac{m_{l}(\mathbf{c})}{l}} t_{i, \mathbf{c} \backslash l} u_{\mathbf{c}}, \quad \tilde{f}_{i l} u=\sum_{\mathbf{c} \in \mathcal{C}_{i}} \sqrt{\frac{l}{m_{l}(\mathbf{c})+1}} t_{i, \mathbf{c} \cup l} u_{\mathbf{c}},
$$

where $m_{l}(\mathbf{c})=\#\left\{k \mid c_{k}=l\right\}$, and $\mathbf{c} \cup l$ stands for the partition $\left(l, c_{1}, \cdots, c_{r}\right)$.

Remark 2.6. Note that the square roots appear in the above definition. So we need to consider an extension $\mathbb{F}$ of $\mathbb{Q}$ that contains all the necessary square roots in Example 2.5 and in Example 2.7. (See [B16, Remark 3.12].)

Let $\mathbb{A}_{0}=\{f \in \mathbb{F}(q) \mid f$ is regular at $q=0\}$ and let $L(\infty)$ be the $\mathbb{A}_{0}$-submodule of $U^{-}$ generated by the elements of the form $\widetilde{f}_{i_{1}, l_{1}} \cdots \tilde{f}_{i_{r}, l_{r}} \mathbf{1}$ for $r \geq 0$ and $\left(i_{k}, l_{k}\right) \in I^{\infty}$. Set

$$
B(\infty)=\left\{\widetilde{f}_{i_{1}, l_{1}} \cdots \widetilde{f}_{i_{r}, l_{r}} \mathbf{1} \bmod q L(\infty) \mid r \geq 0,\left(i_{k}, l_{k}\right) \in I^{\infty}\right\} \subseteq L(\infty) / q L(\infty) .
$$

For $b=\widetilde{f}_{i_{1}, l_{1}} \cdots \widetilde{f}_{i_{r}, l_{r}} \mathbf{1}$, we define

$$
\begin{aligned}
& \operatorname{wt}(b)=-\left(l_{1} \alpha_{i_{1}}+\cdots+l_{r} \alpha_{i_{r}}\right), \\
& \epsilon_{i}(b)= \begin{cases}\max \left\{k \geq 0 \mid \widetilde{e}_{i}^{k} b \neq 0\right\} & \text { for } i \in I^{\mathrm{re}}, \\
0 & \text { for } i \in I^{\mathrm{im}},\end{cases} \\
& \phi_{i}(b)=\epsilon_{i}(b)+\left\langle h_{i}, \operatorname{wt}(b)\right\rangle \text { for any } i \in I .
\end{aligned}
$$

Then $B(\infty)$ becomes an abstract crystal. 
Note that 1 is the only element in $B(\infty)$ of weight 0 and is annihilated by all $\widetilde{e}_{i l}$ for $(i, l) \in I^{\infty}$. Moreover, $B(\infty)$ is connected (as a directed graph).

Example 2.7. For a dominant integral weight $\lambda \in P^{+}$, let $V(\lambda)=U_{q}(\mathfrak{g}) v_{\lambda}$ be the irreducible highest weight module with highest weight $\lambda$ and highest weight vector $v_{\lambda}$.

Let $v \in V(\lambda)$. If $i \in I^{\mathrm{re}}, v$ can be uniquely written as

$$
v=\sum_{k \geq 0} f_{i}^{(k)} v_{k}
$$

where $f_{i}^{(k)}=f_{i}^{k} /[k]_{i}$ ! and $e_{i} v_{k}=0$ for all $k \geq 0$, and if $i \in I^{\text {im }}, v$ can be written uniquely as

$$
v=\sum_{\mathbf{c} \in \mathcal{C}_{i}} t_{i, \mathbf{c}} v_{\mathbf{c}}
$$

where $\mathbf{c}=\left(c_{1}, \ldots, c_{r}\right)$ is a composition or a partition in $\mathcal{C}_{i}$ and $e_{i l} v_{\mathbf{c}}=0$ for all $l \geq 1$. The Kashiwara operators are defined in a similar manner as in Example 2.5.

Let $L(\lambda)$ be the $\mathbb{A}_{0}$-submodule of $V(\lambda)$ generated by the elements of the form $\tilde{f}_{i_{1}, l_{1}} \cdots \tilde{f}_{i_{r}, l_{r}} v_{\lambda}$ for $r \geq 0$ and $\left(i_{k}, l_{k}\right) \in I^{\infty}$. Set

$$
B(\lambda)=\left\{\widetilde{f}_{i_{1}, l_{1}} \cdots \widetilde{f}_{i_{r}, l_{r}} v_{\lambda} \bmod q L(\lambda) \mid r \geq 0,\left(i_{k}, l_{k}\right) \in I^{\infty}\right\} \backslash\{0\} \subseteq L(\lambda) / q L(\lambda) .
$$

For $b=\widetilde{f}_{i_{1}, l_{1}} \cdots \widetilde{f}_{i_{r}, l_{r}} v_{\lambda}$, we define

$$
\begin{aligned}
& \operatorname{wt}(b)=\lambda-\left(l_{1} \alpha_{i_{1}}+\cdots+l_{r} \alpha_{i_{r}}\right), \\
& \epsilon_{i}(b)= \begin{cases}\max \left\{k \geq 0 \mid \widetilde{e}_{i}^{k} b \neq 0\right\} & \text { for } i \in I^{\mathrm{re}}, \\
0 & \text { for } i \in I^{\mathrm{im}},\end{cases} \\
& \phi_{i}(b)=\epsilon_{i}(b)+\left\langle h_{i}, \operatorname{wt}(b)\right\rangle \text { for any } i \in I .
\end{aligned}
$$

Then $B(\lambda)$ becomes an abstract crystal. Note that $v_{\lambda}$ is the only element in $B(\lambda)$ of weight $\lambda$ and is annihilated by all $\widetilde{e}_{i l}$ for $(i, l) \in I^{\infty}$. Moreover, $B(\lambda)$ is connected.

\section{Example 2.8.}


(a) For $\lambda \in P$, set $T_{\lambda}=\left\{t_{\lambda}\right\}$ and define

$$
\operatorname{wt}\left(t_{\lambda}\right)=\lambda, \quad \epsilon_{i}\left(t_{\lambda}\right)=\phi_{i}\left(t_{\lambda}\right)=-\infty, \quad \tilde{e}_{i l}\left(t_{\lambda}\right)=\widetilde{f}_{i l}\left(t_{\lambda}\right)=0
$$

for all $i \in I$ and $l \geq 1$. Then $T_{\lambda}$ is an abstract crystal.

(b) Let $C=\{c\}$ and define

$$
\operatorname{wt}(c)=0, \quad \epsilon_{i}(c)=\phi_{i}(c)=0, \quad \widetilde{e}_{i l}(c)=\widetilde{f}_{i l}(c)=0
$$

for all $i \in I$ and $l \geq 1$. Then $C$ is an abstract crystal which is isomorphic to $B(0)$.

As was proved in [JKK05], we have the following proposition which describes the relation between the crystals $B(\infty)$ and $B(\lambda)$.

Proposition 2.9. For every $\lambda \in P^{+}$, there exists an injective map $\pi_{\lambda}: B(\lambda) \rightarrow B(\infty)$ such that

(i) $\pi_{\lambda}\left(v_{\lambda}\right)=1$,

(ii) for all $(i, l) \in I^{\infty}$ and $b \in B(\lambda)$ with $\widetilde{f}_{i l}(b) \neq 0, \pi_{\lambda} \widetilde{f}_{i l}(b)=\widetilde{f}_{i l} \pi_{\lambda}(b)$,

(iii) for all $(i, l) \in I^{\infty}$ and $b \in B(\lambda), \pi_{\lambda} \widetilde{e}_{i l}(b)=\widetilde{e}_{i l} \pi_{\lambda}(b)$,

(iv) for all $i \in I$ and $b \in B(\lambda), \operatorname{wt}\left(\pi_{\lambda}(b)\right)=\operatorname{wt}(b)-\lambda, \quad \epsilon_{i}\left(\pi_{\lambda}(b)\right)=\epsilon_{i}(b)$.

Remark 2.10. The map $\pi_{\lambda}$ is not a crystal embedding because it does not preserve the functions wt and $\phi_{i}(i \in I)$.

The following example provides a very important class of abstract crystals $B_{i}(i \in I)$ called the elementary crystals.

\section{Example 2.11.}

(a) If $i \in I^{\mathrm{re}}$, let $B_{i}=\{(l) \mid l \geq 0\}$ and define

$$
\begin{aligned}
& \operatorname{wt}((l))=-l \alpha_{i}, \\
& \epsilon_{i}((l))=l, \quad \phi_{i}((l))=-l, \quad \epsilon_{j}((l))=\phi_{j}((l))=-\infty \text { for } j \neq i, \\
& \widetilde{e}_{i}((l))=(l-1), \quad \widetilde{f}_{i}((l))=(l+1), \\
& \widetilde{e}_{j k}((l))=\widetilde{f}_{j k}((l))=0 \text { for } j \neq i .
\end{aligned}
$$


Then $B_{i}$ is an abstract crystal. The crystal graph of $B_{i}$ is given in Figure 1 .

Figure 1

$$
(0) \stackrel{i}{\longrightarrow}(1) \stackrel{i}{\longrightarrow}(2) \stackrel{i}{\longrightarrow} \cdots
$$

We understand $(l)=0$ for $l<0$.

(b) For $i \in I^{\text {im }} \backslash I^{\text {iso }}$, let $B_{i}=\left\{\mathbf{c}=\left(c_{1}, \ldots, c_{r}\right) \mid r \geq 0\right.$, c is a composition $\}$ and define

$$
\begin{aligned}
& \operatorname{wt}(\mathbf{c})=-|\mathbf{c}| \alpha_{i}, \quad \epsilon_{i}(\mathbf{c})=0, \quad \phi_{i}(\mathbf{c})=-|\mathbf{c}| a_{i i}, \\
& \epsilon_{j}(\mathbf{c})=\phi_{j}(\mathbf{c})=-\infty \text { for } j \neq i, \\
& \widetilde{e}_{i l}(\mathbf{c})= \begin{cases}\mathbf{c} \backslash l=\left(c_{2}, \ldots, c_{r}\right) & \text { if } c_{1}=l . \\
0 & \text { otherwise, }\end{cases} \\
& \widetilde{f}_{i l}(\mathbf{c})=(l, \mathbf{c})=\left(l, c_{1}, \ldots, c_{r}\right), \\
& \widetilde{e}_{j k}(\mathbf{c})=\widetilde{f}_{j k}(\mathbf{c})=0 \text { for } j \neq i .
\end{aligned}
$$

Then $B_{i}$ is an abstract crystal. The crystal graph of $B_{i}$ is given in Figure 2 .

Figure 2

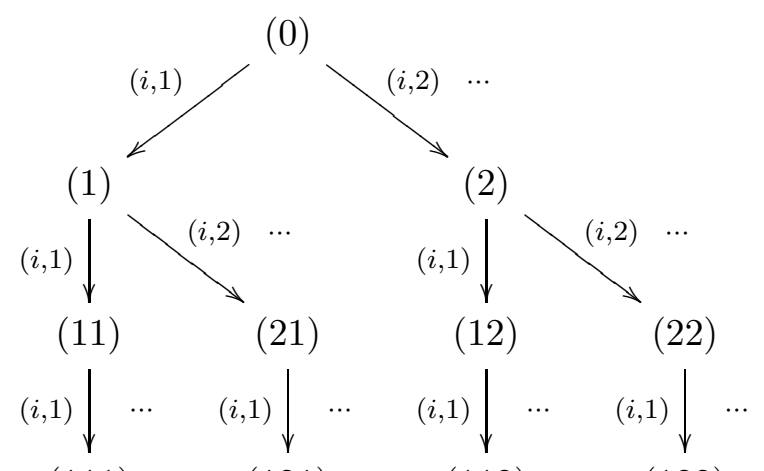

(111)

(c) For $i \in I^{\text {iso }}$, let $B_{i}=\left\{\mathbf{c}=\left(c_{1}, \ldots, c_{r}\right) \mid r \geq 0\right.$, c is a partition $\}$ and define 


$$
\begin{aligned}
& \operatorname{wt}(\mathbf{c})=-|\mathbf{c}| \alpha_{i}, \quad \epsilon_{i}(\mathbf{c})=\phi_{i}(\mathbf{c})=0, \\
& \epsilon_{j}(\mathbf{c})=\phi_{j}(\mathbf{c})=-\infty \text { for } j \neq i, \\
& \widetilde{e}_{i l}(\mathbf{c})= \begin{cases}\mathbf{c} \backslash\{l\} & \text { if } l \text { is a part of } \mathbf{c}, \\
0 & \text { otherwise, }\end{cases} \\
& \widetilde{f}_{i l}(\mathbf{c})=\mathbf{c} \cup l=\left(l, c_{1}, \ldots, c_{r}\right), \\
& \widetilde{e}_{j k}(\mathbf{c})=\widetilde{f}_{j k}(\mathbf{c})=0 \text { for } j \neq i .
\end{aligned}
$$

Then $B_{i}$ is an abstract crystal. A part of the crystal graph of $B_{i}$ is given in Figure 3 .

Figure 3

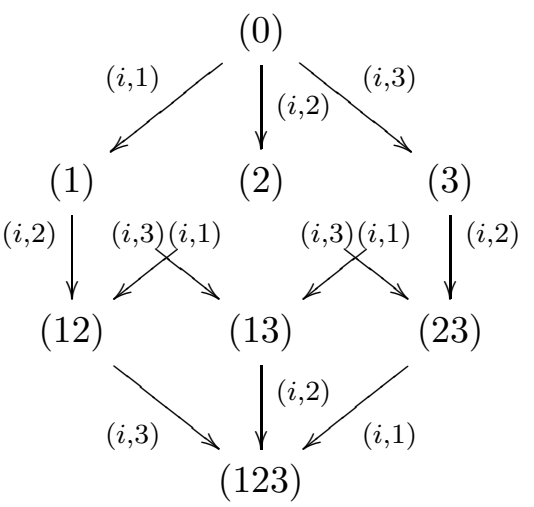

We will write $\mathbf{c}_{i}$ for $\mathbf{c} \in B_{i}$ when we would like to emphasize the index $i$.

Definition 2.12. An abstract crystal $B$ is normal if

$$
\epsilon_{i}(b)=0, \quad \phi_{i}(b) \geq 0 \text { for all } i \in I^{\mathrm{im}}, b \in B .
$$

Remark 2.13. The crystals $B(\infty), B(\lambda)\left(\lambda \in P^{+}\right)$and $C$ are normal, while $T_{\lambda}(\lambda \in P)$ and $B_{i}(i \in I)$ are not normal. 


\section{Tensor PRODUCt OF CRYSTALS}

Let $B_{1}, B_{2}$ be abstract crystals and let $B_{1} \otimes B_{2}=\left\{b_{1} \otimes b_{2} \mid b_{1} \in B_{1}, b_{2} \in B_{2}\right\}$ (as a set). Define the maps wt, $\epsilon_{i}, \phi_{i}(i \in I), \widetilde{e}_{i l}, \widetilde{f}_{i l}\left((i, l) \in I^{\infty}\right)$ as follows.

$$
\begin{aligned}
& \mathrm{wt}\left(b_{1} \otimes b_{2}\right)=\operatorname{wt}\left(b_{1}\right)+\mathrm{wt}\left(b_{2}\right), \\
& \epsilon_{i}\left(b_{1} \otimes b_{2}\right)=\max \left(\epsilon_{i}\left(b_{1}\right), \epsilon_{i}\left(b_{2}\right)-\left\langle h_{i}, \operatorname{wt}\left(b_{1}\right)\right\rangle\right), \\
& \phi_{i}\left(b_{1} \otimes b_{2}\right)=\max \left(\phi_{i}\left(b_{1}\right)+\left\langle h_{i}, \operatorname{wt}\left(b_{2}\right)\right\rangle, \phi_{i}\left(b_{2}\right)\right),
\end{aligned}
$$

If $i \in I^{\mathrm{re}}$,

$$
\begin{aligned}
& \widetilde{e}_{i}\left(b_{1} \otimes b_{2}\right)= \begin{cases}\widetilde{e}_{i} b_{1} \otimes b_{2} & \text { if } \phi_{i}\left(b_{1}\right) \geq \epsilon_{i}\left(b_{2}\right), \\
b_{1} \otimes \widetilde{e}_{i} b_{2} & \text { if } \phi_{i}\left(b_{1}\right)<\epsilon_{i}\left(b_{2}\right),\end{cases} \\
& \widetilde{f}_{i}\left(b_{1} \otimes b_{2}\right)= \begin{cases}\widetilde{f}_{i} b_{1} \otimes b_{2} & \text { if } \phi_{i}\left(b_{1}\right)>\epsilon_{i}\left(b_{2}\right), \\
b_{1} \otimes \widetilde{f}_{i} b_{2} & \text { if } \phi_{i}\left(b_{1}\right) \leq \epsilon_{i}\left(b_{2}\right) .\end{cases}
\end{aligned}
$$

If $i \in I^{\mathrm{im}}$,

$$
\begin{aligned}
& \widetilde{e}_{i l}\left(b_{1} \otimes b_{2}\right)= \begin{cases}\widetilde{e}_{i l} b_{1} \otimes b_{2} & \text { if } \phi_{i}\left(b_{1}\right)>\epsilon_{i}\left(b_{2}\right)-l a_{i i}, \\
0 & \text { if } \epsilon_{i}\left(b_{2}\right)<\phi_{i}\left(b_{1}\right) \leq \epsilon_{i}\left(b_{2}\right)-l a_{i i}, \\
b_{1} \otimes \widetilde{e}_{i l} b_{2} & \text { if } \phi_{i}\left(b_{1}\right) \leq \epsilon_{i}\left(b_{2}\right),\end{cases} \\
& \widetilde{f}_{i l}\left(b_{1} \otimes b_{2}\right)= \begin{cases}\widetilde{f}_{i l} b_{1} \otimes b_{2} & \text { if } \phi_{i}\left(b_{1}\right)>\epsilon_{i}\left(b_{2}\right), \\
b_{1} \otimes \widetilde{f}_{i l} b_{2} & \text { if } \phi_{i}\left(b_{1}\right) \leq \epsilon_{i}\left(b_{2}\right) .\end{cases}
\end{aligned}
$$

Note that if $a_{i i}=0$, we have $\widetilde{e}_{i l}\left(b_{1} \otimes b_{2}\right)=\widetilde{e}_{i l} b_{1} \otimes b_{2}$ when $\phi_{i}\left(b_{1}\right)>\epsilon_{i}\left(b_{2}\right)$, and $\widetilde{e}_{i l}\left(b_{1} \otimes b_{2}\right)=b_{1} \otimes \widetilde{e}_{i l} b_{2}$ when $\phi_{i}\left(b_{1}\right) \leq \epsilon_{i}\left(b_{2}\right)$.

Proposition 3.1. The set $B_{1} \otimes B_{2}$ together with the maps wt, $\epsilon_{i}, \phi_{i}(i \in I), \widetilde{e}_{i l}, \widetilde{f}_{i l}$ $\left((i, l) \in I^{\infty}\right)$ is an abstract crystal.

Proof. It is clear that the conditions (i), (ii) and (iii) in Definition 2.1 hold. If $i \in I^{\mathrm{re}}$, it was shown in [Kas93] that all the conditions are satisfied. For the condition (vii), if 
$\phi_{i}\left(b_{1} \otimes b_{2}\right)=-\infty$, since

$$
\phi_{i}\left(b_{1} \otimes b_{2}\right)=\max \left(\phi_{i}\left(b_{1}\right)+\left\langle h_{i}, \operatorname{wt}\left(b_{2}\right)\right\rangle, \phi_{i}\left(b_{2}\right)\right)
$$

we have $\phi_{i}\left(b_{1}\right)=\phi_{i}\left(b_{2}\right)=-\infty$. Thus

$$
\widetilde{e}_{i l}\left(b_{1} \otimes b_{2}\right)=\widetilde{f}_{i l}\left(b_{1} \otimes b_{2}\right)=0 .
$$

From now on, we will assume that $i \in I^{\mathrm{im}}, l \geq 1$. In this case, we don't have to check the condition (v). Hence we have only to verify the conditions (iv) and (vi).

Suppose $\widetilde{f}_{i l}\left(b_{1} \otimes b_{2}\right)=b_{1}^{\prime} \otimes b_{2}^{\prime}$.

If $\phi_{i}\left(b_{1}\right)>\epsilon_{i}\left(b_{2}\right)$, then $\widetilde{f}_{i l}\left(b_{1} \otimes b_{2}\right)=\widetilde{f}_{i l} b_{1} \otimes b_{2}=b_{1}^{\prime} \otimes b_{2}^{\prime}$. Thus

$$
\phi_{i}\left(b_{1}^{\prime}\right)=\phi_{i}\left(\widetilde{f}_{i l} b_{1}\right)=\phi_{i}\left(b_{1}\right)-l a_{i i}>\epsilon_{i}\left(b_{2}\right)-l a_{i i}=\epsilon_{i}\left(b_{2}^{\prime}\right)-l a_{i i},
$$

which implies $\widetilde{e}_{i l}\left(b_{1}^{\prime} \otimes b_{2}^{\prime}\right)=\widetilde{e}_{i l} b_{1}^{\prime} \otimes b_{2}^{\prime}=b_{1} \otimes b_{2}$.

If, $\phi_{i}\left(b_{1}\right) \leq \epsilon_{i}\left(b_{2}\right)$, then $\widetilde{f}_{i l}\left(b_{1} \otimes b_{2}\right)=b_{1} \otimes \widetilde{f}_{i l} b_{2}=b_{1}^{\prime} \otimes b_{2}^{\prime}$. Note that

$$
\phi_{i}\left(b_{1}^{\prime}\right)=\phi_{i}\left(b_{1}\right) \leq \epsilon_{i}\left(b_{2}\right)=\epsilon_{i}\left(\tilde{f}_{i l} b_{2}\right)=\epsilon_{i}\left(b_{2}^{\prime}\right) .
$$

Thus $\widetilde{e}_{i l}\left(b_{1}^{\prime} \otimes b_{2}^{\prime}\right)=b_{1}^{\prime} \otimes \widetilde{e}_{i l} b_{2}^{\prime}=b_{1} \otimes b_{2}$.

Conversely, suppose $\widetilde{e}_{i l}\left(b_{1}^{\prime} \otimes b_{2}^{\prime}\right)=b_{1} \otimes b_{2}$. Since $\widetilde{e}_{i l}\left(b_{1}^{\prime} \otimes b_{2}^{\prime}\right) \neq 0$, we don't have to consider the case $\epsilon_{i}\left(b_{2}^{\prime}\right)<\phi_{i}\left(b_{1}^{\prime}\right) \leq \epsilon_{i}\left(b_{2}^{\prime}\right)-l a_{i i}$.

If $\phi_{i}\left(b_{1}^{\prime}\right)>\epsilon_{i}\left(b_{2}^{\prime}\right)-l a_{i i}$, we have $\widetilde{e}_{i l}\left(b_{1}^{\prime} \otimes b_{2}^{\prime}\right)=\widetilde{e}_{i l} b_{1}^{\prime} \otimes b_{2}^{\prime}$. Note that

$$
\phi_{i}\left(b_{1}\right)=\phi_{i}\left(\widetilde{e}_{i l} b_{1}^{\prime}\right)=\phi_{i}\left(b_{1}^{\prime}\right)+l a_{i i}>\epsilon_{i}\left(b_{2}^{\prime}\right)=\epsilon_{i}\left(b_{2}\right) .
$$

Hence $\phi_{i}\left(b_{1}\right)>\epsilon_{i}\left(b_{2}\right)$ and we obtain $\widetilde{f}_{i l}\left(b_{1} \otimes b_{2}\right)=\widetilde{f}_{i l} b_{1} \otimes b_{2}=b_{1}^{\prime} \otimes b_{2}^{\prime}$.

If $\phi_{i}\left(b_{1}^{\prime}\right) \leq \epsilon_{i}\left(b_{2}^{\prime}\right)$, then $\widetilde{e}_{i l}\left(b_{1}^{\prime} \otimes b_{2}^{\prime}\right)=b_{1}^{\prime} \otimes \widetilde{e}_{i l} b_{2}^{\prime}$ and we have

$$
\phi_{i}\left(b_{1}\right)=\phi_{i}\left(b_{1}^{\prime}\right) \leq \epsilon_{i}\left(b_{2}^{\prime}\right)=\epsilon_{i}\left(\widetilde{e}_{i l} b_{2}^{\prime}\right)=\epsilon_{i}\left(b_{2}\right),
$$

which gives $\tilde{f}_{i l}\left(b_{1} \otimes b_{2}\right)=b_{1} \otimes \widetilde{f}_{i l} b_{2}=b_{1}^{\prime} \otimes b_{2}^{\prime}$. Hence the condition (iv) is verified.

To verify the condition (vi), let $b_{1} \otimes b_{2} \in B_{1} \otimes B_{2}$ such that $\widetilde{e}_{i l}\left(b_{1} \otimes b_{2}\right) \neq 0$ for all $l \geq 1$. 
If $\phi_{i}\left(b_{1}\right)>\epsilon_{i}\left(b_{2}\right)-l a_{i i}$, then $\widetilde{e}_{i l}\left(b_{1} \otimes b_{2}\right)=\widetilde{e}_{i l} b_{1} \otimes b_{2}$ and hence we get

$$
\begin{aligned}
& \epsilon_{i}\left(\widetilde{e}_{i l}\left(b_{1} \otimes b_{2}\right)\right)=\epsilon_{i}\left(\widetilde{e}_{i l} b_{1} \otimes b_{2}\right) \\
&= \max \left(\epsilon_{i}\left(\widetilde{e}_{i l} b_{1}\right), \epsilon_{i}\left(b_{2}\right)-\left\langle h_{i}, \operatorname{wt}\left(\widetilde{e}_{i l} b_{1}\right)\right\rangle\right) \\
&=\max \left(\epsilon_{i}\left(b_{1}\right), \epsilon_{i}\left(b_{2}\right)-\left\langle h_{i}, \operatorname{wt}\left(b_{1}\right)+l \alpha_{i}\right\rangle\right) \\
&=\max \left(\epsilon_{i}\left(b_{1}\right), \epsilon\left(b_{2}\right)-\left\langle h_{i}, \operatorname{wt}\left(b_{1}\right)\right\rangle-l a_{i i}\right) \\
&=\epsilon_{i}\left(b_{1}\right)=\epsilon_{i}\left(b_{1} \otimes b_{2}\right), \\
& \phi_{i}\left(\widetilde{e}_{i l}\left(b_{1} \otimes b_{2}\right)\right)=\phi_{i}\left(\widetilde{e}_{i l} b_{1} \otimes b_{2}\right) \\
&=\max \left(\phi_{i}\left(\widetilde{e}_{i l} b_{1}\right)+\left\langle h_{i}, \operatorname{wt}\left(b_{2}\right)\right\rangle, \phi_{i}\left(b_{2}\right)\right) \\
&=\max \left(\phi_{i}\left(b_{1}\right)+l a_{i i}+\phi_{i}\left(b_{2}\right)-\epsilon_{i}\left(b_{2}\right), \phi_{i}\left(b_{2}\right)\right) \\
&=\phi_{i}\left(b_{1}\right)+l a_{i i}+\phi_{i}\left(b_{2}\right)-\epsilon_{i}\left(b_{2}\right)=\phi_{i}\left(b_{1} \otimes b_{2}\right)+l a_{i i} .
\end{aligned}
$$

If $\phi_{i}\left(b_{1}\right) \leq \epsilon_{i}\left(b_{2}\right)$, then $\widetilde{e}_{i l}\left(b_{1} \otimes b_{2}\right)=b_{1} \otimes \widetilde{e}_{i l} b_{2}$ and hence we get

$$
\begin{aligned}
\epsilon_{i}\left(\widetilde { e } _ { i l } \left(b_{1}\right.\right. & \left.\left.\otimes b_{2}\right)\right)=\epsilon_{i}\left(b_{1} \otimes \widetilde{e}_{i l} b_{2}\right) \\
& =\max \left(\epsilon_{i}\left(b_{1}\right), \epsilon_{i}\left(\widetilde{e}_{i l} b_{2}\right)-\left\langle h_{i}, \operatorname{wt}\left(b_{1}\right)\right\rangle\right) \\
& =\max \left(\epsilon_{i}\left(b_{1}\right), \epsilon_{i}\left(b_{2}\right)-\left\langle h_{i}, \operatorname{wt}\left(b_{1}\right)\right\rangle\right)=\epsilon_{i}\left(b_{1} \otimes b_{2}\right), \\
\phi_{i}\left(\widetilde { e } _ { i l } \left(b_{1}\right.\right. & \left.\left.\otimes b_{2}\right)\right)=\phi_{i}\left(b_{1} \otimes \widetilde{e}_{i l} b_{2}\right) \\
& =\max \left(\phi_{i}\left(b_{1}\right)+\left\langle h_{i}, \operatorname{wt}\left(\widetilde{e}_{i l} b_{2}\right)\right\rangle, \phi_{i}\left(\widetilde{e}_{i l} b_{2}\right)\right) \\
& =\max \left(\phi_{i}\left(b_{1}\right)+\left\langle h_{i}, \operatorname{wt}\left(b_{2}\right)\right\rangle+l a_{i i}, \phi_{i}\left(b_{2}\right)+l a_{i i}\right) \\
& =\max \left(\phi_{i}\left(b_{1}\right)+\left\langle h_{i}, \operatorname{wt}\left(b_{2}\right)\right\rangle, \phi_{i}\left(b_{2}\right)\right)+l a_{i i} \\
& =\phi_{i}\left(b_{1} \otimes b_{2}\right)+l a_{i i} .
\end{aligned}
$$

By a similar argument, we can verify the condition (vi) for the Kashiwara operators $\tilde{f}_{i l}$ 's.

Corollary 3.2. If $B_{1}$ and $B_{2}$ are normal crystals, then $B_{1} \otimes B_{2}$ is also a normal crystal. 
Proof. For $i \in I^{\mathrm{im}}$, it is easy to see that

$$
\begin{aligned}
\epsilon_{i}\left(b_{1} \otimes b_{2}\right) & =\max \left(\epsilon_{i}\left(b_{1}\right), \epsilon_{i}\left(b_{2}\right)-\left\langle h_{i}, \operatorname{wt}\left(b_{1}\right)\right\rangle\right) \\
& =\max \left(0,-\phi_{i}\left(b_{1}\right)\right)=0, \\
\phi_{i}\left(b_{1} \otimes b_{2}\right) & =\max \left(\phi_{i}\left(b_{1}\right)+\left\langle h_{i}, \operatorname{wt}\left(b_{2}\right)\right\rangle, \phi_{i}\left(b_{2}\right)\right) \\
& =\max \left(\phi_{i}\left(b_{1}\right)+\phi_{i}\left(b_{2}\right), \phi_{i}\left(b_{2}\right)\right) \geq 0,
\end{aligned}
$$

as desired.

Corollary 3.3. For $\lambda, \mu \in P^{+}$, there exists a unique strict crystal embedding

$$
\Phi_{\lambda, \mu}: B(\lambda+\mu) \rightarrow B(\lambda) \otimes B(\mu) \text { given by } v_{\lambda+\mu} \mapsto v_{\lambda} \otimes v_{\mu} .
$$

Proof. By the tensor product rule, $v_{\lambda} \otimes v_{\mu}$ is the only element of weight $\lambda+\mu$ in $B(\lambda) \otimes$ $B(\mu)$. Moreover, it is annihilated by all $\widetilde{e}_{i l}$ 's. Hence the connected component of $B(\lambda) \otimes$ $B(\mu)$ containing $v_{\lambda} \otimes v_{\mu}$ is the full subcrystal of $B(\lambda) \otimes B(\mu)$ which is isomorphic to $B(\lambda+\mu)$.

In the following proposition, we will show that the tensor product of crystals satisfies the associativity law.

Proposition 3.4. Let $B_{i}(i=1,2,3)$ be abstract crystals. Then there exists a unique crystal isomorphism

$$
\Psi:\left(B_{1} \otimes B_{2}\right) \otimes B_{3} \stackrel{\sim}{\longrightarrow} B_{1} \otimes\left(B_{2} \otimes B_{3}\right)
$$

given by $\left.\left(b_{1} \otimes b_{2}\right) \otimes b_{3}\right) \mapsto b_{1} \otimes\left(b_{2} \otimes b_{3}\right)$, where $b_{i} \in B_{i}(i=1,2,3)$.

Proof. It is clear that $\Psi$ is a bijection and preserves the function wt. We shall show that $\Psi$ preserves $\epsilon_{i}, \phi_{i}(i \in I)$ and commutes with $\widetilde{f}_{i l}\left((i, l) \in I^{\infty}\right)$. As we have seen in (2.3), since $\Psi$ is a bijection, we don't have to check the commutativity with $\widetilde{e}_{i l}$ 's.

Let $b=\left(b_{1} \otimes b_{2}\right) \otimes b_{3}$ and $b^{\prime}=\Psi(b)=b_{1} \otimes\left(b_{2} \otimes b_{3}\right)$. Our proof will be divided into the following cases.

Case 1: $\phi_{i}\left(b_{1} \otimes b_{2}\right)>\epsilon_{i}\left(b_{3}\right)$. 
(1) First, assume that $\phi_{i}\left(b_{1}\right)>\epsilon_{i}\left(b_{2}\right)$. In this case, we have

$$
\phi_{i}\left(b_{1} \otimes b_{2}\right)=\phi_{i}\left(b_{1}\right)+\phi_{i}\left(b_{2}\right)-\epsilon_{i}\left(b_{2}\right)>\epsilon\left(b_{3}\right), \quad \epsilon_{i}\left(b_{1} \otimes b_{2}\right)=\epsilon_{i}\left(b_{1}\right),
$$

which yields

$$
\phi_{i}\left(b_{1}\right)+\phi_{i}\left(b_{2}\right)>\epsilon_{i}\left(b_{2}\right)+\epsilon_{i}\left(b_{3}\right) .
$$

Thus we obtain

$$
\begin{aligned}
\phi_{i}(b) & =\phi_{i}\left(\left(b_{1} \otimes b_{2}\right) \otimes b_{3}\right)=\phi_{i}\left(b_{1} \otimes b_{2}\right)+\phi_{i}\left(b_{3}\right)-\epsilon_{i}\left(b_{3}\right) \\
& =\phi_{i}\left(b_{1}\right)+\phi_{i}\left(b_{2}\right)+\phi_{i}\left(b_{3}\right)-\epsilon_{i}\left(b_{2}\right)-\epsilon_{i}\left(b_{3}\right), \\
\epsilon_{i}(b) & =\epsilon_{i}\left(\left(b_{1} \otimes b_{2}\right) \otimes b_{3}\right)=\epsilon_{i}\left(b_{1} \otimes b_{2}\right)=\epsilon_{i}\left(b_{1}\right), \\
\widetilde{f}_{i l}(b) & =\widetilde{f}_{i l}\left(\left(b_{1} \otimes b_{2}\right) \otimes b_{3}\right)=\widetilde{f}_{i l}\left(b_{1} \otimes b_{2}\right) \otimes b_{3}=\left(\widetilde{f}_{i l} b_{1} \otimes b_{2}\right) \otimes b_{3} .
\end{aligned}
$$

On the other hand, to deal with $b^{\prime}$, we compare $\phi_{i}\left(b_{1}\right)$ and $\epsilon_{i}\left(b_{2} \otimes b_{3}\right)$ and obtain

$$
\begin{aligned}
\phi_{i}\left(b^{\prime}\right) & =\phi_{i}\left(b_{1} \otimes\left(b_{2} \otimes b_{3}\right)\right) \\
& =\phi_{i}\left(b_{1}\right)+\phi_{i}\left(b_{2}\right)+\phi_{i}\left(b_{3}\right)-\epsilon_{i}\left(b_{2}\right)-\epsilon_{i}\left(b_{3}\right), \\
\epsilon_{i}\left(b^{\prime}\right) & =\epsilon_{i}\left(b_{1} \otimes\left(b_{2} \otimes b_{3}\right)\right)=\epsilon_{i}\left(b_{1}\right), \\
\tilde{f}_{i l}\left(b^{\prime}\right) & =\widetilde{f}_{i l}\left(b_{1} \otimes\left(b_{2} \otimes b_{3}\right)\right)=\widetilde{f}_{i l} b_{1} \otimes\left(b_{2} \otimes b_{3}\right) .
\end{aligned}
$$

(2) Next, if $\phi_{i}\left(b_{1}\right) \leq \epsilon_{i}\left(b_{2}\right)$, by a similar calculation, we obtain

$$
\begin{aligned}
\phi_{i}(b) & =\phi_{i}\left(\left(b_{1} \otimes b_{2}\right) \otimes b_{3}\right)=\phi_{i}\left(b_{2}\right)+\phi_{i}\left(b_{3}\right)-\epsilon_{i}\left(b_{3}\right), \\
\epsilon_{i}(b) & =\epsilon_{i}\left(\left(b_{1} \otimes b_{2}\right) \otimes b_{3}\right)=\epsilon_{i}\left(b_{1}\right)+\epsilon_{i}\left(b_{2}\right)-\phi_{i}\left(b_{1}\right), \\
\tilde{f}_{i l}(b) & =\widetilde{f}_{i l}\left(\left(b_{1} \otimes b_{2}\right) \otimes b_{3}\right)=\left(b_{1} \otimes \widetilde{f}_{i l} b_{2}\right) \otimes b_{3},
\end{aligned}
$$

and

$$
\begin{aligned}
\phi_{i}\left(b^{\prime}\right) & =\phi_{i}\left(b_{1} \otimes\left(b_{2} \otimes b_{3}\right)\right)=\phi_{i}\left(b_{2}\right)+\phi_{i}\left(b_{3}\right)-\epsilon_{i}\left(b_{3}\right), \\
\epsilon_{i}\left(b^{\prime}\right) & =\epsilon_{i}\left(b_{1} \otimes\left(b_{2} \otimes b_{3}\right)\right)=\epsilon_{i}\left(b_{1}\right)+\epsilon_{i}\left(b_{2}\right)-\phi_{i}\left(b_{1}\right), \\
\tilde{f}_{i l}\left(b^{\prime}\right) & =\widetilde{f}_{i l}\left(b_{1} \otimes\left(b_{2} \otimes b_{3}\right)\right)=b_{1} \otimes\left(\widetilde{f}_{i l} b_{2} \otimes b_{3}\right) .
\end{aligned}
$$

Case 2: $\phi_{i}\left(b_{1} \otimes b_{2}\right) \leq \epsilon_{i}\left(b_{3}\right)$.

In this case, by comparing $\phi_{i}\left(b_{1}\right)$ and $\epsilon_{i}\left(b_{2} \otimes b_{3}\right)$, we obtain 


$$
\begin{aligned}
\phi_{i}(b) & =\phi_{i}\left(\left(b_{1} \otimes b_{2}\right) \otimes b_{3}\right)=\phi_{i}\left(b_{3}\right), \\
\epsilon_{i}(b) & =\epsilon_{i}\left(\left(b_{1} \otimes b_{2}\right) \otimes b_{3}\right)=\epsilon_{i}\left(b_{1}\right)+\epsilon_{i}\left(b_{2}\right)+\epsilon_{i}\left(b_{3}\right)-\phi_{i}\left(b_{1}\right)-\phi_{i}\left(b_{2}\right), \\
\widetilde{f}_{i l}(b) & =\widetilde{f}_{i l}\left(\left(b_{1} \otimes b_{2}\right) \otimes b_{3}\right)=\left(b_{1} \otimes b_{2}\right) \otimes \widetilde{f}_{i l} b_{3},
\end{aligned}
$$

and

$$
\begin{aligned}
\phi_{i}\left(b^{\prime}\right) & =\phi_{i}\left(b_{1} \otimes\left(b_{2} \otimes b_{3}\right)\right)=\phi_{i}\left(b_{3}\right), \\
\epsilon_{i}\left(b^{\prime}\right) & =\epsilon_{i}\left(b_{1} \otimes\left(b_{2} \otimes b_{3}\right)\right)=\epsilon_{i}\left(b_{1}\right)+\epsilon_{i}\left(b_{2}\right)+\epsilon_{i}\left(b_{3}\right)-\phi_{i}\left(b_{1}\right)-\phi_{i}\left(b_{2}\right), \\
\widetilde{f}_{i l}\left(b^{\prime}\right) & =\widetilde{f}_{i l}\left(b_{1} \otimes\left(b_{2} \otimes b_{3}\right)\right)=b_{1} \otimes\left(b_{2} \otimes \widetilde{f}_{i l} b_{3}\right) .
\end{aligned}
$$

Thus we have proved all of our assertions.

\section{Crystal embedding theorem}

In this section, we prove one of the main results in this paper, the crystal embedding theorem for quantum Borcherds-Bozec algebras.

Theorem 4.1. For $i \in I$, there is a unique strict crystal embedding

$$
\Psi_{i}: B(\infty) \hookrightarrow B(\infty) \otimes B_{i} \text { given by } \mathbf{1} \mapsto \mathbf{1} \otimes(0)_{i}
$$

Proof. Since there is only one vector of weight 0 in $B(\infty) \otimes B_{i}$, which is $\mathbf{1} \otimes(0)_{i}, \Psi_{i}$ should send $\mathbf{1}$ to $\mathbf{1} \otimes(0)_{i}$ if $\Psi_{i}$ exists, because it is a crystal morphism.

Let $b=\widetilde{f}_{i_{1}, l_{1}} \cdots \widetilde{f}_{i_{r}, l_{r}} \mathbf{1} \in B(\infty)$. Choose $\lambda \in P^{+}$such that

(i) $\left\langle h_{j}, \lambda\right\rangle \gg 0$ for all $j \in I$,

(ii) $b \in \operatorname{Im} \pi_{\lambda}$,

where $\pi_{\lambda}: B(\lambda) \rightarrow B(\infty)$ is the injective map given in Proposition 2.9.

Set $b_{\lambda}=\widetilde{f}_{i_{1}, l_{1}} \cdots \widetilde{f}_{i_{r}, l_{r}} v_{\lambda} \in B(\lambda)$ so that $\pi_{\lambda}\left(b_{\lambda}\right)=b \in B(\infty)$.

Let $l=\left\langle h_{i}, \lambda\right\rangle$ and $\mu=\lambda-l \Lambda_{i} \in P^{+}$. Then $\left\langle h_{i}, \mu\right\rangle=0$ and by Corollary 3.3 there exists a unique strict crystal embedding

$$
\Phi_{\mu, l \Lambda_{i}}: B(\lambda) \rightarrow B(\mu) \otimes B\left(l \Lambda_{i}\right) \text { given by } v_{\lambda} \mapsto v_{\mu} \otimes v_{l \Lambda_{i}} .
$$

We will show that 
(1) $\Phi_{\mu, l \Lambda_{i}}\left(b_{\lambda}\right)=b^{\prime} \otimes \widetilde{f}_{i, \mathbf{c}} v_{l \Lambda_{i}}$ for some $b^{\prime} \in B(\mu), \mathbf{c} \in B_{i}$,

(2) $\pi_{\mu}\left(b^{\prime}\right) \otimes \mathbf{c} \in B(\infty) \otimes B_{i}$ does not depend on the choice of $\lambda \gg 0$.

Here, if $i \in I^{\mathrm{re}}$, then $\mathbf{c} \in B_{i}$ is a non-negative integer $c$ and we understand $\widetilde{f}_{i, \mathbf{c}}=\widetilde{f}_{i}^{c}$.

Once our claims are proved, we will get a well-defined map

$$
\Psi_{i}: B(\infty) \rightarrow B(\infty) \otimes B_{i} \text { given by } b \mapsto \pi_{\mu}\left(b^{\prime}\right) \otimes \mathbf{c} .
$$

We will prove our assertions by induction on $r \geq 0$. When $r=0$, our assertion is obvious. Assume that $r>0$ and our assertion is true for $r-1$.

Set $b_{1}=\tilde{f}_{i_{2}, l_{2}} \cdots \tilde{f}_{i_{r}, l_{r}} v_{\lambda}$. By our induction hypothesis, we have

(1a) $\Phi_{\mu, l \Lambda_{i}}\left(b_{1}\right)=b_{1}^{\prime} \otimes \widetilde{f}_{i, \mathbf{c}^{\prime}} v_{l \Lambda_{i}}$ for some $b_{1}^{\prime} \in B(\mu), \mathbf{c}^{\prime} \in B_{i}$,

(2a) $\pi_{\mu}\left(b_{1}^{\prime}\right) \otimes \mathbf{c}^{\prime} \in B(\infty) \otimes B_{i}$ does not depend on the choice of $\lambda$.

Therefore it suffices to show that

(1b) $\widetilde{f}_{i_{1}, l_{1}}\left(b_{1}^{\prime} \otimes \widetilde{f}_{i, \mathbf{c}^{\prime}} v_{l \Lambda_{i}}\right)=b^{\prime} \otimes \widetilde{f}_{i, \mathbf{c}} v_{l \Lambda_{i}}$ for some $b^{\prime} \in B(\mu), \mathbf{c} \in B_{i}$.

(2b) $\tilde{f}_{i_{1}, l_{1}}\left(\pi_{\mu}\left(b_{1}^{\prime}\right) \otimes \mathbf{c}^{\prime}\right)=\pi_{\mu}\left(b^{\prime}\right) \otimes \mathbf{c}$.

If $i_{1}=i$, then $\left\langle h_{i}, \mu\right\rangle=0$ and hence

$$
\begin{aligned}
& \phi_{i}\left(b_{1}^{\prime}\right)=\phi_{i}\left(\pi_{\mu}\left(b_{1}^{\prime}\right)\right), \\
& \epsilon_{i}\left(\tilde{f}_{i, \mathbf{c}^{\prime}} v_{l \Lambda_{i}}\right)=\epsilon_{i}\left(\mathbf{c}^{\prime}\right)= \begin{cases}c^{\prime} & \text { if } i \in I^{\mathrm{re}}, \\
0 & \text { if } i \in I^{\mathrm{im}}\end{cases}
\end{aligned}
$$

Therefore, $\widetilde{f}_{i_{1}, l_{1}}$ acts on the 1st component (resp. 2nd component) of (1b) if and only if it acts on the 1st component (resp. 2nd component) of (2b), which proves our claim.

If $i_{1} \neq i$, then $\epsilon_{i_{1}}\left(\mathbf{c}^{\prime}\right)=-\infty$ and

$$
\phi_{i_{1}}\left(b_{1}^{\prime}\right)=\phi_{i_{1}}\left(\pi_{\mu}\left(b_{1}^{\prime}\right)\right)+\mu\left(h_{i_{1}}\right) \gg 0=\epsilon_{i_{1}}\left(\widetilde{f}_{i, \mathbf{c}^{\prime}} v_{l \Lambda_{i}}\right) .
$$

Hence $\widetilde{f}_{i_{1}, l_{1}}$ acts on the 1 st component of $(1 \mathrm{~b})$ and $(2 \mathrm{~b})$, which yields our claim.

It is straightforward to verify that $\Psi_{i}$ is a strict crystal morphism. 
Our next goal is to provide a characterization of the crystals $B(\infty)$ and $B(\lambda)\left(\lambda \in P^{+}\right)$. To this end, we introduce an important family of crystals arising from tensor products of elementary crystals.

Let $\mathbf{i}=\left(i_{1}, i_{2}, \ldots\right)$ be an infinite sequence of indices in $I$ such that every $i \in I$ appears infinitely many times. Set

$$
B_{\mathbf{i}}=\left\{b=\cdots \otimes \mathbf{c}_{k} \otimes \cdots \otimes \mathbf{c}_{1} \mid \mathbf{c}_{k} \in B_{i_{k}}, \mathbf{c}_{k}=(0)_{i_{k}} \text { for } k \gg 0\right\}
$$

Using the tensor product rule, there is a crystal structure on $B_{\mathbf{i}}$ defined as follows. (See [JKKS07] for a more rigorous and detailed treatment.)

Let $b=\cdots \otimes \mathbf{c}_{k} \otimes \cdots \otimes \mathbf{c}_{1}$, where $\mathbf{c}_{k} \in B_{i_{k}}$. Then we define

$$
\begin{aligned}
\operatorname{wt}(b) & =-\sum_{k \geq 1}\left|\mathbf{c}_{k}\right| \alpha_{i_{k}}, \\
\epsilon_{i}(b) & = \begin{cases}\max \left\{c_{k}+\sum_{p>k} c_{p} a_{i, i_{p}} \mid k \geq 1, i_{k}=i\right\} & \text { if } i \in I^{\mathrm{re}}, \\
0 & \text { if } i \in I^{\mathrm{im}},\end{cases} \\
\phi_{i}(b) & = \begin{cases}\max \left\{-c_{k}-\sum_{1 \leq p<k} c_{p} a_{i, i_{p}} \mid k \geq 1, i_{k}=i\right\} & \text { if } i \in I^{\mathrm{re}}, \\
-\sum_{k \geq 1}\left|\mathbf{c}_{k}\right| a_{i, i_{k}} & \text { if } i \in I^{\mathrm{im}} .\end{cases}
\end{aligned}
$$

To define the Kashiwara operators, we first assume $i \in I^{\text {re }}$ and let $s$ (resp. $t$ ) be the largest (resp. smallest) integer $k \geq 1$ such that

(i) $i_{k}=i$,

(ii) $c_{k}+\sum_{p>k} c_{p} a_{i, i_{p}}=\epsilon_{i}(b)$.

Then we define

$$
\begin{aligned}
& \tilde{e}_{i}(b)= \begin{cases}\cdots \otimes \mathbf{c}_{s+1} \otimes\left(c_{s}-1\right) \otimes \mathbf{c}_{s-1} \otimes \cdots \otimes \mathbf{c}_{1} & \text { if } \epsilon_{i}(b)>0, \\
0 & \text { otherwise, }\end{cases} \\
& \widetilde{f}_{i}(b)=\cdots \otimes \mathbf{c}_{t+1} \otimes\left(c_{t}+1\right) \otimes \mathbf{c}_{t-1} \otimes \cdots \otimes \mathbf{c}_{1} .
\end{aligned}
$$

Suppose $i \in I^{\mathrm{im}}$ and let $r$ be the smallest integer $k \geq 1$ such that

(i) $i_{k}=i$, 
(ii) $\sum_{p>k}\left|\mathbf{c}_{p}\right| a_{i, i_{p}}=0$.

Then we define

$$
\widetilde{f}_{i, l}(b)=\cdots \otimes \mathbf{c}_{r+1} \otimes\left(l, \mathbf{c}_{r}\right) \otimes \mathbf{c}_{r-1} \otimes \cdots \otimes \mathbf{c}_{1} .
$$

Assume further that

(i) the 1st component of $\mathbf{c}_{r}$ is $l$ or $\mathbf{c}_{r}$ is a partition having $l$ as a part,

(ii) $\sum_{s<p \leq r}\left|\mathbf{c}_{p}\right| a_{i, i_{p}}<l a_{i i}$ for any $s$ with $i_{s}=i$ and $1 \leq s<r$.

In this case, we define

$$
\widetilde{e}_{i, l}(b)=\cdots \otimes \mathbf{c}_{r+1} \otimes\left(\mathbf{c}_{r} \backslash l\right) \otimes \mathbf{c}_{r-1} \otimes \cdots \otimes \mathbf{c}_{1} .
$$

Otherwise, we define $\widetilde{e}_{i l}(b)=0$.

For each $N \geq 1$, along the sequence $\mathbf{i}=\left(i_{1}, i_{2}, \ldots\right)$, we apply the crystal embedding theorem repeatedly to get a strict crystal embedding

$$
\Psi^{(N)}: B(\infty) \hookrightarrow B(\infty) \otimes B_{i_{1}} \hookrightarrow B(\infty) \otimes B_{i_{2}} \otimes B_{i_{1}} \hookrightarrow \cdots \hookrightarrow B(\infty) \otimes B_{i_{N}} \otimes \cdots \otimes B_{i_{1}} .
$$

For each $b \in B(\infty)$, it is easy to see that there exists some $N \geq 1$ satisfying

$$
\Psi^{(N)}(b)=\mathbf{1} \otimes \mathbf{c}_{N} \otimes \cdots \otimes \mathbf{c}_{1} \in B(\infty) \otimes B_{i_{N}} \otimes \cdots \otimes B_{i_{1}} .
$$

Hence to each $b \in B(\infty)$, one can associate a unique element

$$
b_{\mathbf{i}}=\cdots \otimes(0)_{i_{N+1}} \otimes \mathbf{c}_{N} \otimes \cdots \otimes \mathbf{c}_{1} \in B_{\mathbf{i}}
$$

which yields a strict crystal embedding

$$
\Psi_{\mathbf{i}}: B(\infty) \hookrightarrow B_{\mathbf{i}}
$$

Therefore $B(\infty)$ is isomorphic to the connected component of $B_{\mathbf{i}}$ containing the element $(0)_{\mathbf{i}}=\cdots \otimes(0)_{i_{N}} \otimes \cdots \otimes(0)_{i_{1}}$. In particular, $\mathbf{1}$ is mapped onto $(0)_{\mathbf{i}}$.

We will now give a characterization of $B(\infty)$ as an application of the crystal embedding theorem.

Theorem 4.2. Let $B$ be a crystal satisfying the following conditions.

(i) $\operatorname{wt}(B) \subset Q^{-}$,

(ii) there exists an element $b_{0} \in B$ such that $\operatorname{wt}\left(b_{0}\right)=0$, 
(iii) for any $b \neq b_{0}$, there is some $(i, l) \in I^{\infty}$ such that $\widetilde{e}_{i l} b \neq 0$,

(iv) for each $i \in I$, there exists a strict crystal embedding $\Psi_{i}: B \hookrightarrow B \otimes B_{i}$.

Then there exists a crystal isomorphism

$$
B \stackrel{\sim}{\longrightarrow} B(\infty) \text { given by } b_{0} \mapsto \mathbf{1}
$$

Proof. Note that for any element $b \in B$ with wt $(b)=0$, we have $\widetilde{e}_{i l}(b)=0$ for all $(i, l) \in I^{\infty}$, for otherwise, $\operatorname{wt}\left(\widetilde{e}_{i l} b\right)=l \alpha_{i} \notin Q^{-}$. Hence by the condition (iii), if $\operatorname{wt}(b)=0$, then $b=b_{0}$, which implies $b_{0}$ is the only element in $B$ such that $\operatorname{wt}(b)=0$. It follows that the crystal embedding $\Psi_{i}$ maps $b_{0}$ to $b_{0} \otimes(0)_{i}$.

Take an infinite sequence $\mathbf{i}=\left(i_{1}, i_{2}, \ldots\right)$ such that every $i \in I$ appears infinitely many times. Then for each $N \geq 1$, we obtain a strict embedding

$$
\Psi^{(N)}: B \hookrightarrow B \otimes B_{i_{1}} \hookrightarrow B \otimes B_{i_{2}} \otimes B_{i_{1}} \hookrightarrow \cdots \hookrightarrow B \otimes B_{i_{N}} \otimes \cdots \otimes B_{i_{1}}
$$

sending $b_{0}$ to $b_{0} \otimes(0)_{i_{N}} \otimes \cdots \otimes(0)_{i_{1}}$.

As we have seen in the discussion above the theorem, for each $b \in B$, there exists $N \geq 1$ such that

$$
\Psi^{(N)}(b)=b_{0} \otimes \mathbf{c}_{N} \otimes \cdots \otimes \mathbf{c}_{1},
$$

which yields a strict crystal embedding

$$
B \hookrightarrow B(\mathbf{i}) \text { given by } b \mapsto \cdots \otimes(0)_{i_{N+1}} \otimes \mathbf{c}_{N} \otimes \cdots \otimes \mathbf{c}_{1}\left(\mathbf{c}_{k} \in B_{i_{k}}\right) .
$$

Thus $B$ is isomorphic to the connected component of $B_{\mathbf{i}}$ containing the element $(0)_{\mathbf{i}}=$ $\cdots \otimes(0)_{i_{N}} \otimes \cdots \otimes(0)_{i_{1}}$, which implies $B$ is isomorphic to $B(\infty)$ and $b_{0}$ is mapped to 1.

Let us turn to the crystal $B(\lambda)\left(\lambda \in P^{+}\right)$. The properties of the crystals $T_{\lambda}=\left\{t_{\lambda}\right\}$ and $C=\{c\}$ introduced in Example 2.8 will play an important role in our characterization of $B(\lambda)$.

For $\lambda \in P^{+}$, consider the injective map

$$
\psi_{\lambda}: B(\lambda) \rightarrow B(\infty) \otimes T_{\lambda} \text { given by } b \mapsto \pi_{\lambda}(b) \otimes t_{\lambda},
$$


where $\pi_{\lambda}: B(\lambda) \rightarrow B(\infty)$ is the injective map given in Proposition 2.9. One can immediately see that $\psi_{\lambda}$ preserves the function wt. Since $\epsilon_{i}\left(t_{\lambda}\right)=\phi_{i}\left(t_{\lambda}\right)=-\infty$, for $b \in B(\lambda)$, we have

$$
\begin{aligned}
\epsilon_{i}(b) & =\epsilon_{i}\left(\pi_{\lambda}(b)\right)=\epsilon_{i}\left(\pi_{\lambda}(b) \otimes t_{\lambda}\right), \\
\phi_{i}(b) & =\epsilon_{i}(b)+\left\langle h_{i}, \operatorname{wt}(b)\right\rangle \\
& =\epsilon_{i}\left(\pi_{\lambda}(b) \otimes t_{\lambda}\right)+\left\langle h_{i}, \operatorname{wt}\left(\pi_{\lambda}(b) \otimes t_{\lambda}\right)\right\rangle \\
& =\phi_{i}\left(\pi_{\lambda}(b) \otimes t_{\lambda}\right) .
\end{aligned}
$$

Hence $\psi_{\lambda}$ preserves $\epsilon_{i}, \phi_{i}(i \in I)$.

If $b \in B(\lambda)$ and $\widetilde{f}_{i l} b \in B(\lambda)$, Proposition 2.9 yields

$$
\tilde{f}_{i l}\left(\psi_{\lambda}(b)\right)=\tilde{f}_{i l}\left(\pi_{\lambda}(b) \otimes t_{\lambda}\right)=\tilde{f}_{i l}\left(\pi_{\lambda}(b)\right) \otimes t_{\lambda}=\pi_{\lambda}\left(\tilde{f}_{i l} b\right) \otimes t_{\lambda}=\psi_{\lambda}\left(\tilde{f}_{i l} b\right) .
$$

Moreover,

$$
\widetilde{e}_{i l}\left(\psi_{\lambda}(b)\right)=\widetilde{e}_{i l}\left(\pi_{\lambda}(b) \otimes t_{\lambda}\right)=\widetilde{e}_{i l}\left(\pi_{\lambda}(b)\right) \otimes t_{\lambda}=\pi_{\lambda}\left(\widetilde{e}_{i l} b\right) \otimes t_{\lambda}=\psi_{\lambda}\left(\widetilde{e}_{i l} b\right) .
$$

Thus $\psi_{\lambda}$ is a crystal morphism commuting with all $\widetilde{e}_{i l}$ 's.

Consider the injective map

$$
\iota_{\lambda}: B(\lambda) \rightarrow B(\infty) \otimes T_{\lambda} \otimes C \text { given by } b \mapsto \psi_{\lambda}(b) \otimes c \text {. }
$$

For $b \in B(\lambda)$, we have

$$
\begin{aligned}
\operatorname{wt}\left(\iota_{\lambda}(b)\right) & =\operatorname{wt}\left(\psi_{\lambda}(b)\right)+\operatorname{wt}(c)=\operatorname{wt}(b), \\
\phi_{i}\left(\iota_{\lambda}(b)\right) & =\max \left(\phi_{i}\left(\psi_{\lambda}(b)\right)+\left\langle h_{i}, \operatorname{wt}(c)\right\rangle, \phi_{i}(c)\right) \\
& =\max \left(\phi_{i}(b), 0\right)=\phi_{i}(b), \\
\epsilon_{i}\left(\iota_{\lambda}(b)\right) & =\phi_{i}\left(\iota_{\lambda}(b)\right)-\left\langle h_{i}, \operatorname{wt}\left(\iota_{\lambda}(b)\right)\right\rangle \\
& =\phi_{i}(b)-\left\langle h_{i}, \operatorname{wt}(b)\right\rangle=\epsilon_{i}(b) .
\end{aligned}
$$


Furthermore, since $b \in B(\lambda)$, we have $\phi_{i}\left(\psi_{\lambda}(b)\right)=\phi_{i}(b) \geq 0=\epsilon_{i}(c)$. Thus if $i \in I^{\mathrm{re}}$, then

$$
\begin{aligned}
\widetilde{e}_{i}\left(\iota_{\lambda}(b)\right) & =\widetilde{e}_{i}\left(\psi_{\lambda}(b) \otimes c\right)=\widetilde{e}_{i}\left(\psi_{\lambda}(b)\right) \otimes c \\
& =\psi_{\lambda}\left(\widetilde{e}_{i} b\right) \otimes c=\iota_{\lambda}\left(\widetilde{e}_{i} b\right), \\
\tilde{f}_{i}\left(\iota_{\lambda}(b)\right) & =\widetilde{f}_{i}\left(\psi_{\lambda}(b) \otimes c\right) \\
& = \begin{cases}\widetilde{f}_{i} \psi_{\lambda}(b) \otimes c=\psi_{\lambda}\left(\widetilde{f}_{i} b\right) \otimes c & \text { if } \phi_{i}(b)>0, \\
0 & \text { if } \phi_{i}(b)=0, \\
& =\iota_{\lambda}\left(\widetilde{f}_{i} b\right) .\end{cases}
\end{aligned}
$$

If $i \in I^{\mathrm{im}}$ and $\phi_{i}(b)=\phi_{i}\left(\psi_{\lambda}(b)\right)>-l a_{i i}$, then we have

$$
\widetilde{e}_{i l}\left(\iota_{\lambda}(b)\right)=\widetilde{e}_{i l}\left(\psi_{\lambda}(b) \otimes c\right)=\widetilde{e}_{i l}\left(\psi_{\lambda}(b)\right) \otimes c=\psi_{\lambda}\left(\widetilde{e}_{i l} b\right) \otimes c=\iota_{\lambda}\left(\widetilde{e}_{i l} b\right) .
$$

If $i \in I^{\mathrm{im}}$ and $0<\phi_{i}(b)=\phi_{i}\left(\psi_{\lambda}(b)\right) \leq-l a_{i i}$, then

$$
\widetilde{e}_{i l}\left(\iota_{\lambda}(b)\right)=\widetilde{e}_{i l}\left(\psi_{\lambda}(b) \otimes c\right)=0 .
$$

On the other hand, since $\epsilon_{i}(b)=0$, our condition implies

$$
0<\phi_{i}(b)=\left\langle h_{i}, \operatorname{wt}(b)\right\rangle \leq-l a_{i i} .
$$

By the definition of category $\mathcal{O}_{\text {int }}\left(\left[\mathrm{KK} 19 \mathrm{~b}\right.\right.$, Definition 5.1]), we have $\widetilde{e}_{i l}(b)=0$, which implies

$$
\widetilde{e}_{i l}\left(\iota_{\lambda}(b)\right)=\iota_{\lambda}\left(\widetilde{e}_{i l} b\right)=0
$$

as desired.

If $i \in I^{\mathrm{im}}$ and $\phi_{i}(b)=\phi_{i}\left(\psi_{\lambda}(b)\right)=0$, then $\left\langle h_{i}\right.$, wt $\left.(b)\right\rangle=0$, which implies $\widetilde{e}_{i l}(b)=0$ and

$$
\widetilde{e}_{i l}\left(\iota_{\lambda}(b)\right)=\widetilde{e}_{i l}\left(\psi_{\lambda}(b) \otimes c\right)=\psi_{\lambda}(b) \otimes \widetilde{e}_{i l}(c)=0=\psi_{\lambda}\left(\widetilde{e}_{i l}(b)\right) \otimes c=\iota_{\lambda}\left(\widetilde{e}_{i l}(b)\right) .
$$

For the operators $\widetilde{f}_{i l}$, if $i \in I^{\mathrm{im}}$ and $\phi_{i}(b)=\phi_{i}\left(\psi_{\lambda}(b)\right)>0$, then

$$
\widetilde{f}_{i l}\left(\iota_{\lambda}(b)\right)=\widetilde{f}_{i l}\left(\psi_{\lambda}(b) \otimes c\right)=\widetilde{f}_{i l}\left(\psi_{\lambda}(b)\right) \otimes c=\psi_{\lambda}\left(\widetilde{f}_{i l}(b)\right) \otimes c=\iota_{\lambda}\left(\widetilde{f}_{i l} b\right) .
$$


If $i \in I^{\mathrm{im}}$ and $\phi_{i}(b)=0$, then $\widetilde{f}_{i l}(b)=0$ and hence

$$
\widetilde{f}_{i l}\left(\iota_{\lambda}(b)\right)=\widetilde{f}_{i l}\left(\psi_{\lambda}(b) \otimes c\right)=\psi_{\lambda}(b) \otimes \widetilde{f}_{i l} c=0=\iota_{\lambda}\left(\widetilde{f}_{i l} b\right)
$$

as desired.

Therefore, $\iota_{\lambda}: B(\lambda) \rightarrow B(\infty) \otimes T_{\lambda} \otimes C$ is a strict crystal embedding and obtain the following characterization of $B(\lambda)$.

Theorem 4.3. Let $\lambda \in P^{+}$be a dominant integral weight. Then the crystal $B(\lambda)$ is isomorphic to the connected component of $B(\infty) \otimes T_{\lambda} \otimes C$ containing $\mathbf{1} \otimes t_{\lambda} \otimes c$.

Remark 4.4. (a) As was mentioned at the end of [B16], our characterization can be applied to reproduce Bozec's geometric realization of $B(\infty)$ and $B(\lambda)$ (cf. [KKS09, KKS12]).

(b) Our constuction of $B(\infty)$ and $B(\lambda)$ can also be used directly to the theory of (dual) perfect bases for quantum Borcherds-Bozec algebras.

(c) Our theory will play a crucial role in the categorification of quantum BorcherdsBozec algebras and their highest weight modules via Khovanov-Lauda-Rouquier algebras and their cyclotomic quotients.

\section{REFERENCES}

[Bor88] R. Borcherds, Generalized Kac-Moody algebras, J. Algebra 115 (1988), 501-512.

[B15] T. Bozec, Quivers with loops and perverse sheaves, Math. Ann. 362 (2015), 773-797.

[B16] T. Bozec, Quivers with loops and generalized crystals, Compositio Math. 152 (2016), 1999-2040.

[BSV16] T. Bozec, O. Schiffmann and E. Vasserot, On the number of points of nilpotent quiver varieties over finite fields, arXiv:1701.01797.

[FKKT20] Z. Fan, S.-J. Kang, Y. R. Kim and B. Tong, Classical limit of quantum Borcherds-Bozec algebras, J. Pure Appl. Algebra, 225 (2021), 106502.

[GL93] I. Grojnowski and G. Lusztig, A comparison of bases of quantized enveloping algebras, Contemporary Math., 153 (1993), 11-19.

[HK02] J. Hong and S.-J. Kang, Introduction to Quantum Groups and Crystal Bases, Graduate Studies in Mathematics 42, Amer. Math. Soc., 2002.

[JKK05] K. Jeong, S.-J. Kang and M. Kashiwara, Crystal Bases for quantum generalized Kac-Moody algebras, Proc. Lond. Math. Soc. (3) 90 (2005), 395-438. 
[JKKS07] K. Jeong, S.-J. Kang, M. Kashiwara and D.-U. Shin, Abstract crystals for quantum generalized Kac-Moody algebras, Int. Math. Res. Not. IMRN (2007), no.1. 1-19.

[J95] A. Joseph, Quantum groups and their primitive ideals, Springer-Verlag, Berlin, 1995

[Kac68] V. G. Kac, Simple irreducible graded Lie algebras of finite growth, Math. USSR. Izv. 2 (1968), 1271-1311.

[K19] S.-J. Kang, Borcherds-Bozec algebras, root multiplicities and the Schofield construction, Communications in Contemporary Mathematics 21 (2019), no.3.

[K95] S.-J. Kang, Quantum deformations of generalized Kac-Moody algebras and their modules, J. Algebra (3) 175 (1995), 1041-1066

[KKS09] S.-J. Kang, M. Kashiwara and O. Schiffmann, Geometric construction of crystal bases for quantum generalized Kac-Moody algebras, Adv. Math.222 (2009), 996-1015.

[KKS12] S.-J. Kang, M. Kashiwara and O. Schiffmann, Geometric construction of highest weight crystals for quantum generalized Kac-Moody algebras, Math. Ann. 354 (2012), 193-208.

[KK19b] S.-J. Kang and Y. R. Kim, Quantum Borcherds-Bozec algebras and their integrable representations, J. Pure Appl. Algebra 224 (2020), 106388.

[Kas90] M. Kashiwara, Crystallizing the q-analogue of universal enveloping algebras, Commun. Math. Phys. 133 (1990), 249-260.

[Kas91] M. Kashiwara, On crystal bases of the q-analogue of universal enveloping algebras, Duke Math. J. 63 (1991), 465-516.

[Kas93] M. Kashiwara, The crystal base and Littlemann's refined Demazure character formula, Duke Math. J. 71 (1993), 839-858.

[KS97] M. Kashiwara and Y. Saito, Geometric construction of crystal bases, Duke Math. J. 89 (1997), 9-36.

[L90] G. Lusztig, Canonical bases arising from quantized enveloping algebras, J. Amer. Math. Soc. 3 (1990), 447-498.

[L91] G. Lusztig, Quivers, perverse sheaves, and quantized enveloping algebras, J. Amer. Math. Soc. 4 (1991), no. 2, 365-421.

[L10] G. Lusztig, Introduction to quantum groups, Modern Birkhäuser Classics, Birkhäuser/Springer, New York, 2010

[Moody68] R. V. Moody, A new class of Lie algebras, J. Algebra 10 (1968), 211-230.

[NZ97] T. Nakashima and A. Zelevinsky, Polyhedral realizations of crystal bases for quantized KacMoody algebra, Adv. Math. 131 (1997), 253-278.

[S02] Y. Saito, Crystal bases and quiver varieties, Math. Ann. 324 (2002), 675-688. 
Harbin Engineering University, Harbin, China

Email address: fanz@ksu.edu

Korea Research Institute of Arts and Mathematics, Asan-si, Chungcheongnam-do, 31551 , KOREA

Email address: soccerkang@hotmail.com

Graduate School of Education, Hankuk University of Foreign Studies, Seoul, 02450, KoREA

Email address: rocky777@hufs.ac.kr

Harbin Engineering University, Harbin, China

Email address: tbl_2019@hrbeu.edu.cn 